\title{
Evolución geodinámica pleistocena del valle del río Arlanzón: implicaciones en la formación del sistema endokárstico y los yacimientos al aire libre de la Sierra de Atapuerca (Burgos, España)
}

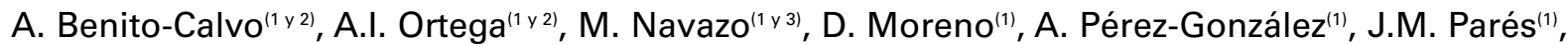
J.M. Bermúdez de Castro(1) y E. Carbonell ${ }^{(4,5 \text { y } 6)}$

(1) Centro Nacional de Investigación sobre la Evolución Humana (CENIEH), Paseo de la Sierra de Atapuerca 3 , E-09002 Burgos, España alfonso.benito@cenieh.es, davinia.moreno@cenieh.es, alfredo.perez@cenieh.es, josep.pares@cenieh.es y josemaria.bermudezdecastro@cenieh.es

(2) Grupo Espeleológico Edelweiss, Paseo del Espolón s/n, E-09071 Burgos, España anaisabel.ortega@cenieh.es

(3) Área de Prehistoria, Departamento de Historia, Geografía y Comunicación, Facultad de Humanidades y Comunicación Universidad de Burgos, Paseo Comendadores s/n (Hospital Militar), E-09001 Burgos, España mnavazo@ubu.es

(4) IPHES, Institut Català de Paleoecología Humana i Evolució Social, C/ Marcelli Domingo s/n Campus Sescelades URV (Edifici W3), E-43007Tarragona, España eudald.carbonell@urv.cat

(5) Universitat Rovira i Virgili (URV), Campus Catalunya, Avinguda de Catalunya 35, E-43002 Tarragona, España. (6) Institute of Vertebrate Palaeontology and Palaeoanthropology (IVPP), Beijing, PR China

\section{RESUMEN}

El valle del río Arlanzón se localiza al NE de la depresión del Duero (Meseta Norte Castellana, España), donde drena el anticlinal de la Sierra de Atapuerca, que presenta un conjunto arqueológico de yacimientos kársticos y al aire libre desde los últimos 1.22 Ma. En el valle se reconocen 14 terrazas fluviales, desde T1 (+92-100 m) hasta T14 $(+2-3 \mathrm{~m})$, desarrolladas desde el Pleistoceno inferior y relacionadas principalmente con estadios isotópicos (Marine Isotopic Stages, MIS). Las dataciones disponibles y las alturas relativas de las terrazas, medidas con datos LiDAR, han permitido reconstruir las fases de encajamiento fluvial, caracterizadas por una aceleración de las tasas de incisión a lo largo del Cuaternario, probablemente relacionado con un levantamiento tectónico. En el sistema endokárstico de la Sierra de Atapuerca, se identificaron tres niveles de conductos freáticos subhorizontales, relacionados espacialmente y cronológicamente con las terrazas T2 (+82-91 m), T3 (+70$78 \mathrm{~m})$ y T4 $(+60-67 \mathrm{~m}) / \mathrm{T5}(+50-58 \mathrm{~m})$ del río Arlanzón. La asociación entre terrazas fluviales y conductos freáticos sugiere un tiempo de formación relativamente corto para los conductos freáticos, separados por largos periodos de encajamientos kársticos vadosos, que se relacionan con las bajas tasas incisión fluvial del río Arlanzón ( $<0.038-0.045 \mathrm{~m} / \mathrm{ka}$ ). En el valle del río Arlanzón, incisiones de $0.061 \mathrm{~m} / \mathrm{ka}$ provocaron la exhumación progresiva de una capa neógena con sílex, cuya erosión y resedimentación en las vertientes de los valles durante diversas fases a lo largo del Pleistoceno medio y superior, generó varias áreas de captación, explotadas por los neandertales durante los MIS 4 y 3.

Palabras clave: endokarst, Pleistoceno, río Arlanzón, Sierra de Atapuerca, terrazas fluviales.

\section{Pleistocene geodynamic evolution of the Arlanzón valley: implications for the formation of the endokarst system} and the open air archaeological sites of the Sierra de Atapuerca (Burgos, España)

\author{
ABSTRACT
}

The Arlanzón river is located in the NE Duero Depression (Castilian Plateau of northern Spain), where it drains the anticlinal ridge of the Sierra de Atapuerca, where a rich record of karstic and open air Palaeolithic archaeological sites are located, spanning the last 1.22 Ma. The geomorphological sequence of this valley is composed 
of fourteen fluvial terraces from T1 $(+92-100 \mathrm{~m})$, to levelT14 $(+2-3 \mathrm{~m})$, ranging from the Early Pleistocene to the Holocene and mainly related to cold MIS. Available chronological data and terrace relative heights extracted from LiDAR data, have allowed the reconstruction of the downcutting phases of the valley, which show an acceleration of the incision rates throughout the Quaternary, probably related to tectonic uplift. In the Sierra de Atapuerca multilevel endokarst system, three sub-horizontal cave levels appear spatially and chronologically related to the Arlanzón fluvial terraces T2 $(+82-91 \mathrm{~m})$, T3 $(+70-78 \mathrm{~m})$ and T4 $(+60-67 \mathrm{~m}) / \mathrm{T} 5(+50-58 \mathrm{~m})$. The association between fluvial terrace base levels and sub-horizontal phreatic caves suggests a relatively short period of time for the formation of these phreatic passages when compared to karstic vadose entrenchments related to the low incision rates of the Arlanzón river $(<0.038-0.045 \mathrm{~m} / \mathrm{ka}$ ). In the valley, the incision of $0.061 \mathrm{~m} / \mathrm{ka} \mathrm{cau}$ sed the progressive exhumation of a neogene limestone layer containing flint, which erosion and successive re-sedimentation phases by alluvial and colluvial processes on the valley slopes during the Middle and Upper Pleistocene caused many wide captation areas of raw material, which were exploited by the Neanderthals during MIS 3 and 4.

Key words: Arlanzón river, endokarst, fluvial terraces, Pleistocene, Sierra de Atapuerca.

\section{ABRIDGED ENGLISH VERSION}

\section{Introduction and methods}

The Arlanzón river is located in the NE Duero Depression (High Plateau of northern Spain, Fig. 1), where it flows along $131.6 \mathrm{~km}$ from the Sierra de la Demanda (San Millán Peak, $2132 \mathrm{~m}$ a.s.l.), to the junction with the Arlanza river in the Duero Neogene Basin, at $750 \mathrm{~m}$ a.s.l. The middle Arlanzón valley drains the anticlinal ridge of the Sierra de Atapuerca (Fig. 1), where a rich record of Palaeolithic archaeological sites are located, spanning the last 1.22 Ma (Arsuaga et al., 2014; Bermúdez de Castro et al., 2013; Carbonell et al., 2008). These sites are located in different environments within a multilevel endokarst system (Ortega et al., 2014, 2013; Ortega, 2009), and in open air places in the valley (Navazo and Carbonell, 2014; Navazo et al., 2011; 2008a y b; Mosquera et al., 2007; Navazo, 2006). The formation and preservation of all these sites were controlled by the dynamics of the Pleistocene base levels of the Arlanzón valley, which were driven, in turn, by the lithological characteristics of the bedrock, the climatic changes and the regional uplift.

The middle Arlanzón valley has been investigated in several studies dealing with its morphogenesis (BenitoCalvo et al., 2008; Benito-Calvo and Pérez-González, 2007; Benito, 2004; Pérez-González et al., 2001; PérezGonzález et al., 1999; Pineda and Arce, 1997; García et al., 1997; Zazo et al., 1983, 1987), the numeric chronology of its Quaternary deposits (Arnold et al., 2013; Moreno et al., 2012; Moreno, 2011; Benito-Calvo et al., 2008), and the palaeolandscape modelling around the Sierra de Atapuerca (Benito-Calvo et al., 2008). Recently, a geomorphological map of the Sierra de Atapuerca has also been published, based on LiDAR data (Benito-Calvo and Pérez-González, 2015).

In this study, we present the geomorphological analysis of the complete Arlanzón valley, updating the identification and geometry of landforms by means of LiDAR data and aerial photographs digital anaglyphs, using GIS (ArcGIS 10.2). Furthermore, we include the geomorphological sequences of the Vena and Pico valleys, tributaries of the middle Aralnzón valley. These valleys together drain the Sierra de Atapuerca. Geomorphological data is used in combination with the available chronological data to correlate the terrace sequence with the Marine Isotope Stages (MIS) and to reconstruct the evolution of the incision rates in this area during the Pleistocene. These analyses are used to explain the geomorphological genesis of the open air sites in the valley, and to analyse the influence of the regional geodynamic evolution in the genesis of the Sierra de Atapuerca endokarst system. The latter in turn is complemented by the research presented by Ortega et al. (in this volume), where the relationships between karstic processes and archaeological site formation in this endokarst are analysed.

\section{Results and discussion}

This study area is located in the junction area between the NE intraplate Iberian Chain (Sierra de la Demanda) and the Duero Cenozoic basin (Fig. 1). The Arlanzón river erodes Palaeozoic materials of the Iberian Massif, carbonated and siliciclastic Mesozoic rocks, Oligocene-Miocene syn-orogenic detritic sediments, and the post-orogenic Neogene succession made up of endorheic lacustrine and alluvial facies.

In this region we have studied the upper, middle and lower Arlanzón valley (Figs. 2-3). The landform sequence in this region of the NE Duero Basin begins with planation surfaces developed during the erosion-sedimentation/uplift cycles during the Neogene (Benito-Calvo and Pérez-González, 2015, 2007). The last planation surface (SE4- Pl, Figs. 3-5) probably developed during the Plio-Quaternary, after the Upper Miocene endorheic 
Benito-Calvo, A., et al., 2018. Evolución geodinámica pleistocena del valle del río Arlanzón... Boletín Geológico y Minero, 129 (1/2): $59-82$

culminant surface and the Duero Basin opening to the Atlantic Ocean. From this surface, the downcutting of the Arlanzon valley has defined a compound rectilinear route, integrated by E-W, NE-SW, NW-SE directions imposed by bedrock alignments (Benito, 2004; Pineda, 1997; Pérez-González, 1994; Zazo et al., 1983). In these rectilinear routes, the Arlanzón valley displays asymmetrical geometries (Figs. 2-4), characterised by more stepped right margins and scarps in the left margins. This asymmetry defines a general trend of the valley to migrate towards the south (in most of the middle Arlanzón valley) and towards the east (lower Arlanzón valley). The exorheic incision of the Arlanzón valley is defined by a fluvial staircase model, which is composed of fourteen fluvial terraces. These fluvial terraces develop from terrace T1, at $+92-100 \mathrm{~m}$ above the current river, to levelT14, at $+2-3 \mathrm{~m}$ above the river. These levels are terraces with 2-4 $m$ of mean thickness (Fig. 5), consisting mainly of alluvial gravel channels, with scarce fine channel facies and overbank deposits (Fig. 6). The terraces are characterised by $A / E / B t$ soil profiles in the high levels, varying to $A / B t$ in the middle and lower levels and profiles $A / C$ in the floodplain (Pérez-González et al., 1999).

Available dating techniques (Arnold et al., 2013; Moreno et al., 2012; Benito-Calvo et al., 2008; Fig. 8), suggest an Early Pleistocene age for T3 $(+70-78 \mathrm{~m})$ and T4 $(+60-67 \mathrm{~m})$, and Middle Pleistocene for T5 $(+50-58 \mathrm{~m})$ until T11 (+12-14 m), which would be close to the Middle-Late Pleistocene boundary. These ages provide a significant correlation between fluvial terrace and cold marine isotope stages (Fig. 8). The ages and relative heights of terraces have allowed the estimation of the incision rates of $0.038 \mathrm{~m} / \mathrm{ka}$ for the end of the Early Pleistocene (T3-T4), $0.045 \mathrm{~m} / \mathrm{ka}$ in the boundary between the Early-Middle Pleistocene, 0.081-0.185 m/ka during the Middle Pleistocene, and around $0.081 \mathrm{~m} / \mathrm{ka}$ for the Late Pleistocene. The highest incision rates would have occurred in the Holocene. This sequence shows an acceleration of the incision rates, which could be explained by regional uplift, as this is observed in other worldwide staircase fluvial systems (Bridgland and Westaway, 2008). Limitations of dating techniques for old terraces do not allow us to estimate the aggradational rates of these fluvial terraces, although comparison with Late Pleistocene terraces and current floodplain development (Thorndycraft and Benito, 2006; Pérez-González et al., 2008) suggest that fluvial terrace sedimentation could develop in relatively short periods of time when compared to the incision period between the terrace levels.

This dynamic has controlled the formation of the Sierra de Atapuerca endokarst system, which is composed mainly of three subhorizontal caves, associated with stable phreatic levels marked by Arlanzón fluvial terraces T2 (+82-91 m), T3 (+70-78 m) and T4 (+60-67 m)/T5 (+50-58 m) (Ortega et al., 2014, 2013; Ortega, 2009). This association suggests a span for the karst formation between a relatively fast formation of the phreatic morphologies in the endokarst during cold stages, separated by long periods when low main incision rates between $<0.038 \mathrm{~m} / \mathrm{ka}$ and $0.045 \mathrm{~m} / \mathrm{ka}$ would have driven the vadose entrenchment described in the endokarst. During most of the Early Pleistocene (between around 2.2 and 1.22-1.13 Ma), the very low incision ratios $<0.038 \mathrm{~m} / \mathrm{ka}$ occurred in the Arlanzón valley near the Sierra de Atapuerca would have kept the base levels at a similar position, generating a dense succession of phreatic levels in the endokarst, which cause the big volumes of the upper endokarst level. Below this level, the phreatic morphology of the middle endokarstic level, developed in the Early Pleistocene, is related to the base levelT3 (+70-78 m) (Ortega et al., 2013, 2014), in a period coinciding with the terrace ESR date $(1.14 \pm 0.13 \mathrm{Ma}$ (Moreno et al., 2012) and the two dates estimated for the base of Elefante site (1.13 $\pm 0.18-1.22 \pm 0.16 \mathrm{Ma}$, Carbonell et al., 2008); all three are statistically indistinguishable (Parés et al., 2016). This level was abandoned at the end of the Early Pleistocene, with fluvial incision rates of $0.038 \mathrm{~m} / \mathrm{ka}$, which drove the vadose entrenchment between the intermediate and the lower endokarst levels (e.g. lower part of Elefante or Doline sites). The lower endokast level is associated with phreatic levels marked by terraces T4 $(+60-67 \mathrm{~m})$ and T5 (+50-58 m), which represent the last known endokarst level, probably between 0.85-0.66 Ma. During the Middle Pleistocene, incison rates increased to $0.061 \mathrm{~m} / \mathrm{ka}$ which caused the abandonment of the lower endokarstic phreatic level. During this period, the wide exhumation of a Neogene limestone layer including flint occurred in the valley. The erosion of flint nodules from this layer, and successive re-sedimentation phases by alluvial and colluvial processes on the valley slopes during the Middle and Upper Pleistocene, formed many wide captation areas of raw material (Figs. 9 and 10). Neanderthal populations took advantage of this during the Upper Pleistocene (Navazo and Carbonell, 2014; Navazo et al., 2011; 2008a and b; Navazo, 2006), where several archaeological sites show reiterative occupation between 70 and 50 ka and coinciding with MIS 3 and 4 (Navazo et al., 2008a; Arnold et al., 2013). At these sites, flint was being exploited where Pleistocene landforms were being eroded by alluvial and gravitational processes.

\section{Introducción}

El río Arlanzón se localiza en el sector NE de la depresión del Duero, donde fluye a lo largo de $131.6 \mathrm{~km}$, desde las estribaciones en los materiales paleozoicos de la sierra de la Demanda (Pico de San Millán, 2132 m s.n.m.), hasta su confluencia con el río Arlanza en posiciones centrales de la cuenca neógena del Duero, a 750 m s.n.m.. En su tramo medio, el río Arlanzón drena el anticlinal de la Sierra de Atapuerca, donde se encuentran numerosos yacimientos paleolíticos, que abarcan al menos los últimos 1.22 Ma (Arsuaga et al., 
2014; Bermúdez de Castro et al., 2013; Carbonell et al., 2008). Estos yacimientos se encuentran en diferentes ambientes dentro de un sistema endokárstico multinivel (Ortega et al., 2014, 2013; Ortega, 2009), y en posiciones al aire libre (Navazo and Carbonell, 2014; Navazo et al., 2011; 2008a and b; Navazo, 2006). La formación y preservación de estos yacimientos han estado controladas por la dinámica pleistocena de los niveles de base del valle del río Arlanzón en función de las características litoestructurales del sustrato, los cambios climáticos cuaternarios y los levantamientos tectónicos regionales.

En este sentido, la evolución del tramo medio del valle del río Arlanzón y su secuencia de terrazas han sido objeto de varios trabajos con la finalidad de investigar su morfogénesis (Benito-Calvo et al., 2008; Benito-Calvo and Pérez-González, 2007; Benito, 2004; Pérez-González et al., 2001; Pérez-González et al., 1999; Pineda y Arce, 1997; García et al., 1997; Zazo et al., 1983, 1987), la cronología numérica de sus depósitos cuaternarios (Arnold et al., 2013; Moreno, 2011; Moreno et al., 2012, Benito-Calvo et al., 2008), o la reconstrucción de paleopaisajes alrededor de la Sierra de Atapuerca (Benito-Calvo et al., 2008). Además, recientemente se ha realizado la cartografía geomorfológica con datos LiDAR de la Sierra de Atapuerca y sus alrededores (Benito-Calvo and PérezGonzález, 2015).

En este trabajo, se presenta el análisis geomorfológico a lo largo del tramo completo del río Arlanzón, actualizando la identificación y geometría de los registros geomorfológicos en base a datos LiDAR y anaglifos digitales. Además, se incluyen las secuencias geomorfológicas de los valles del río Vena y Pico, tributarios menores del río Arlanzón, que en conjunto drenan la Sierra de Atapuerca. Los datos geomorfológicos, junto con los datos cronológicos disponibles, son utilizados para presentar la correlación regional de sus terrazas y su asociación con los estadios isotópicos marinos (MIS). La posición LiDAR de los niveles de terraza es utilizada junto con las cronologías existentes para reconstruir la evolución de las tasas de incisión durante el Pleistoceno. Los datos obtenidos son aplicados para reconstruir la formación de los yacimientos arqueológicos al aire libre, así como para analizar la influencia de la geodinámica regional en la formación del sistema kárstico multinivel de la Sierra de Atapuerca.

\section{Contexto geológico}

La cuenca de drenaje del río Arlanzón presenta un desnivel total de $1381 \mathrm{~m}$, que se distribuyen en los sistemas morfoclimáticos húmedo, semiárido y secocontinental (Benito-Calvo et al., 2009). Durante su recorrido, el río Arlanzón ha excavado su valle en el Macizo Ibérico de la sierra de la Demanda, en su orla mesozoica, y en los sedimentos continentales de la cuenca cenozoica del Duero (Fig. 1). Los materiales paleozoicos están compuestos por metasedimentos cámbricos, ordovícicos y carboníferos, deformados por la orogenia Varisca, cuyas estructuras mayores son fallas de direcciones NE-SO, NO-SE, E-W y el sinclinal SE-NO de Palazuelos-Alarzón (Boquera et al., 1978; Colchen, 1970). Las rocas mesozoicas se distribuyen rodeando al Paleozoico de la sierra de la Demanda (Cabrera et al., 1997; García et al., 1997; Pineda and Arce, 1997; Olivé et al., 1990; Boquera et al., 1978), formando sierras de anticlinales y sinclinales de dirección Ibérica (Fig. 1), donde se distinguen rocas de edad Triásico, Jurásico y Cretácico (facies Weald del Cretácico inferior, depósitos detríticos siliciclásticos del Aptiense-Albiense, y la secuencia carbonatada del Cretácico superior).

En la cuenca cenozoica del Duero, el río Arlanzón drena los depósitos conglomeráticos sinorogénicos del Oligoceno-Mioceno inferior (Pineda and Arce, 1997; Santistéban et al., 1996), y la serie post-orogénica Miocena, principalmente subhorizontal (AlonsoGavilán et al., 2004; Mediavilla et al., 1996; Fig. 1), compuesta por sedimentos lacustres y evaporíticos del Mioceno inferior (facies Dueñas, Villatoro y Cerezo). Por encima se desarrollan depósitos principalmente aluviales del Mioceno medio (facies Santa María del Campo, Tierra de Campos, Pedraja; García et al., 1997; Pineda, 1996; Núñez et al., 1975; Pavón et al., 1973), con desarrollo a techo de procesos edáficos (Mediavilla et al., 1996) o de una capa de calizas astaracienses con nódulos de sílex, deformada en el contacto con la Sierra de Atapuerca (Benito-Calvo et al., 2008).

Estas facies aluviales del Mioceno medio se hayan cubiertas por depósitos del Mioceno superior (Fig. 1), eminentemente lacustres (facies Cuestas), que culminan con las calizas del páramo inferior (Pineda and Arce, 1997; García et al., 1997). La sedimentación endorreica cenozoica de la cuenca del Duero culmina con el ciclo sedimentario del páramo superior (Fig. 1), integrado por unidades de arcillas rojas y arenas, margas blancas y las calizas del páramo superior (Benito and Pérez-González, 2005; García et al., 1997; Núñez et al., 1975; Pavón et al., 1973), de edad estimada en Mioceno superior final (Turoliense superior, yacimiento de Tariego de Cerrato (Pérez et al., 2001). En Mondúbar de San Cibrián, el ciclo sedimentario del páramo superior se encuentra plegado por un sinclinal de dirección SE-NO, donde los estratos presentan buzamientos de 3-4 (García et al., 1997). 


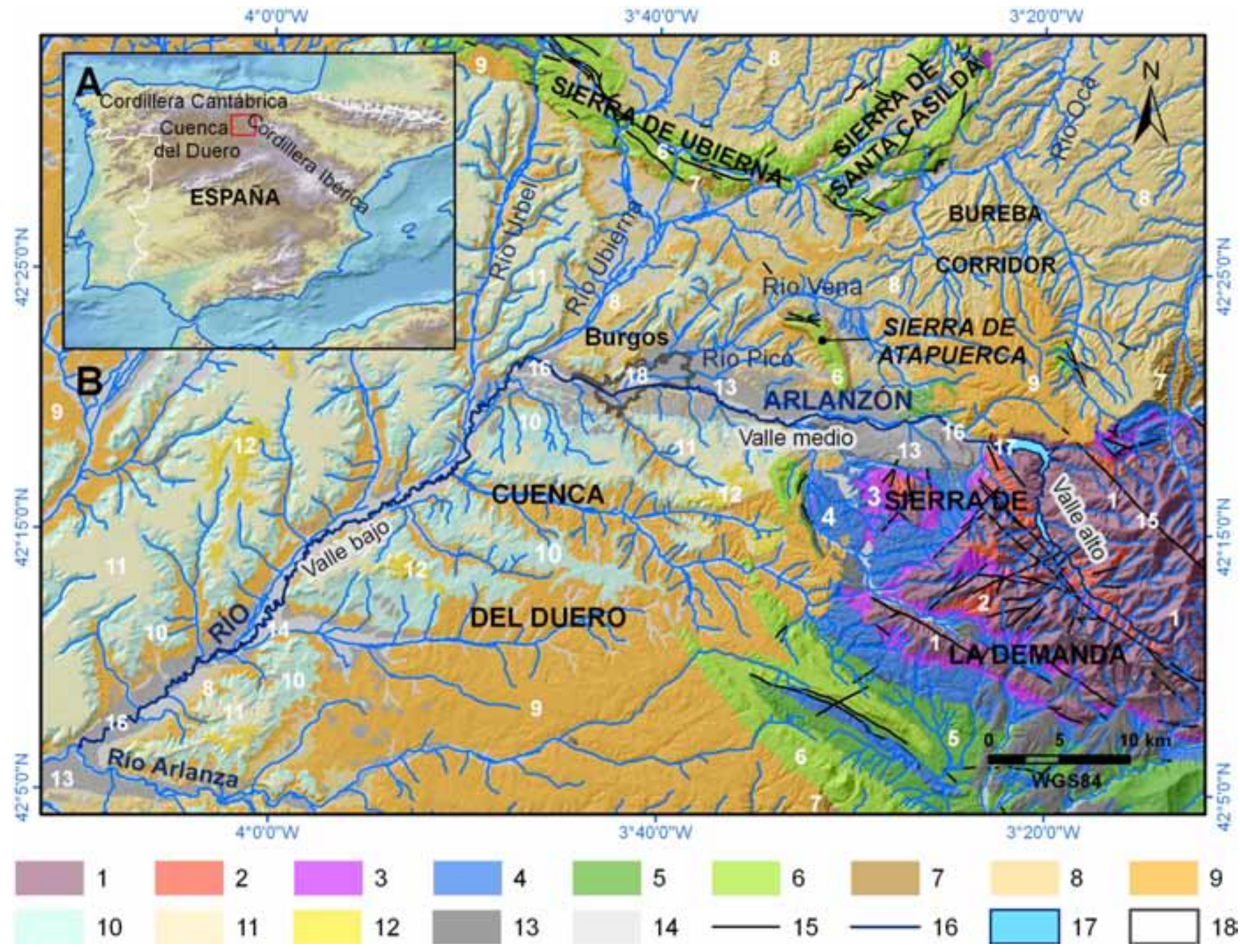

Figura 1. Contexto regional del río Arlanzón. A) Situación del área de estudio en la Península lbérica. B) Mapa geológico regional. Leyenda: 1, Pizarras y cuarcitas (Cámbrico-Ordovícico); 2, Conglomerados, areniscas y lutitas (Carbonífero); 3, Sedimentos siliciclásticos, calizas, arcillas y evaporitas (Triásico); 4, Carbonatos y margas (Jurásico); 5, Conglomerados, areniscas y calizas (Cretácico inferior); 6, Carbonatos y margas (Cretácito superior); 7, Conglomerados, arenas y arcillas (Paleógeno-Mioceno); 8,Margas, arcillas y evaporitas (Mioceno inferior); 9, Gravas, arenas y arcillas, localmente calizas (Mioceno medio, Astaraciense); 10, Margas, arcillas y calizas (Mioceno superior); 11 , Calizas del páramo inferior (Mioceno superior); 12, Sedimentos detríticos y calizas del páramo superior (Mioceno superior, Turoliense); 13, Terrazas fluviales, conos aluviales, coluviones (Pleistoceno-Holoceno); 14, Llanuras aluviales y fondos de valle (Holoceno); 15, Fallas; 16, río Arlanzón; 17, Embalse; 18, Población principal.

Figure 1. Regional framework of the Arlanzón valley. A) Study area in the Iberian Peninsula. B) Regional geological map. Legend: 1 , slate and quarzites (Cambrian-Ordovician); 2, conglomerates, sandstones and shales (Carboniferous); 3, siliciclastic sediments, limestones, clays and evaporites (Triassic); 4, carbonates and marls (Jurassic); 5, conglomerates, sandstones and limestones (Early Cretaceous); 6 , carbonates and marls (Late Cretaceous); 7 , conglomerates, sands and clays (Paleogene-Miocene); 8, marls, clays and evaporites (Early Miocene); 9, gravels, sands, clays and local limestones (Middle Miocene, Astaracian); 10, marls, clays and limestones (Late Miocene); 11, Lower páramo limestones (Late Miocene); 12, detrital sediments and upper páramo limestones (Late Miocene, Turolian); 13, fluvial terraces, alluvial fans, coluvions (Pleistocene-Holocene); 14, floodplains and valley beds (Holocene); 15 faults; 16, Arlanzón River; 17, reservoirs; 18, main city.

Con anterioridad a la instalación de sistemas fluviales escalonados, diversos autores citan la presencia de gravas silíceas y arcillas de tipo raña, asignados al Plioceno o Plio-Cuaternario (García et al., 1997; Mediavilla et al., 1996; Olivé et al. 1990; Zazo et al.
1983, 1987; Boquera et al. 1978; Núñez et al., 1975; Zazo and Goy, 1977), aunque en la mayoría de los casos se encuadran en sistemas escalonados de terrazas fluviales o facies detrícicas de borde neógenas (Benito, 2004). 


\section{Metodología}

El análisis geomorfológico se ha basado en el trabajo de campo y en el análisis cartográfico de diversas bases de datos espaciales. En el trabajo de campo se utilizaron GPS y cámaras fotográficas con GPS integrado, para el registro espacial georreferenciado de formas y depósitos. Respecto a las bases de datos espaciales, se han empleado fotografías aéreas de 1956 (Vuelo Americano, escala 1:33.000), de 19801986 (Vuelo Nacional, escala 1:18.000), y de 2009 (Vuelo digital PNOA, con resoluciones de $25 \mathrm{~cm}$ ). Estos fotogramas han sido transformados en anaglifos digitales georreferenciados para el análisis este- reoscópico del territorio en plataformas SIG (ArcGIS 10.2). Se ha utilizado el Modelo Digital del Terreno MDT05-LiDAR del Instituto Geográfico Nacional para estimar la geometría y posición relativa de las morfologías del terreno, junto con los datos planimétricos y topográficos del Mapa del Territorio Nacional a escala 1:25.000 (MNT25). La interpretación y cartografía del terreno estuvo apoyada por los mapas geológicos 1:50.000 del Instituto Geológico y Minero de España (IGME). Los trabajos geomorfológicos se centraron especialmente en el tramo medio del río Arlanzón, donde se sitúa la Sierra de Atapuerca y sus yacimientos arqueológicos, además de contener la secuencia más completa de terrazas cuaternarias.

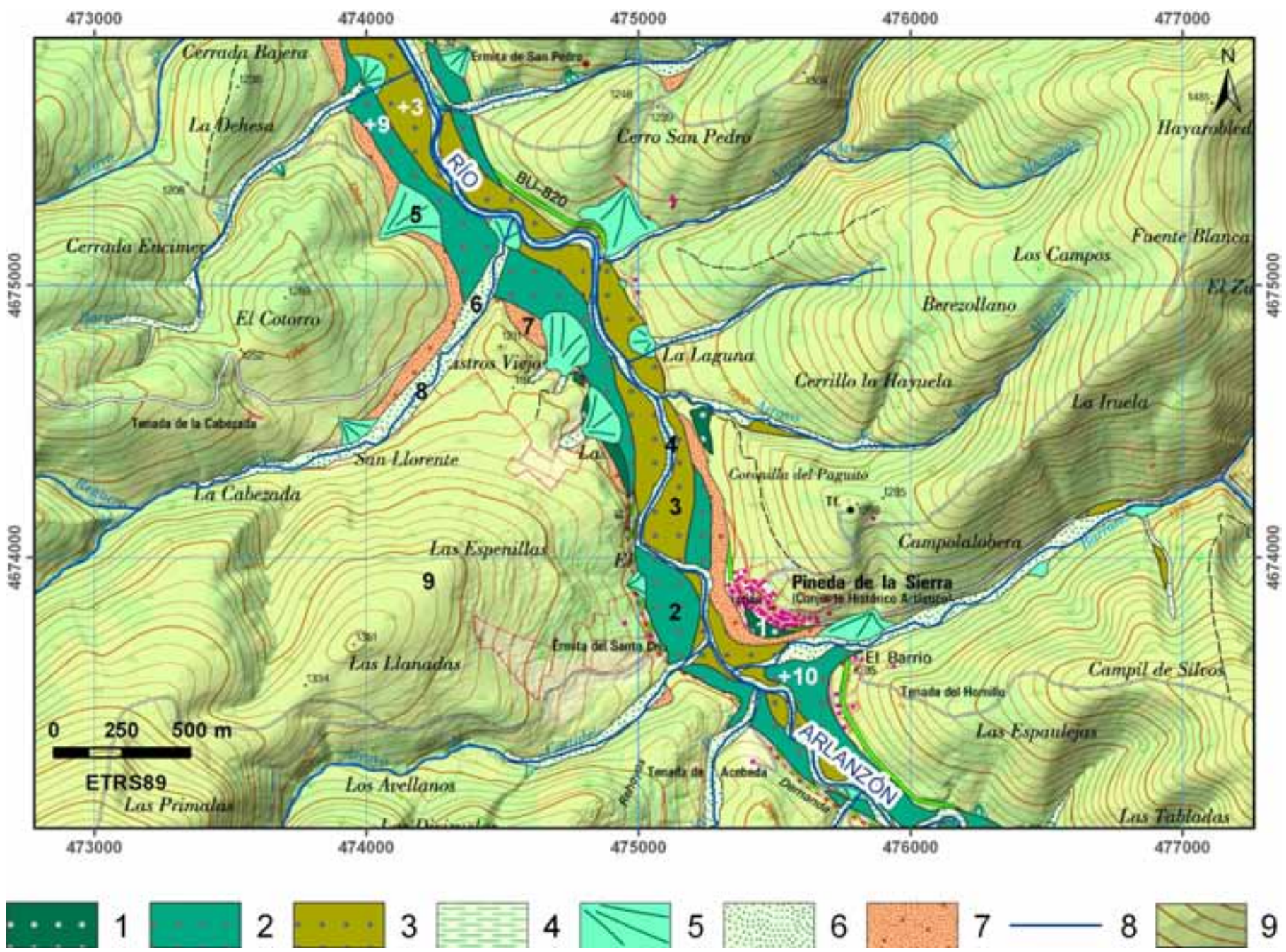

Figura 2. Terrazas fluviales bajas en el área de Pinedo, valle alto del río Arlanzón. Leyenda: 1, Terraza a +12-15 m; 2, Terraza a +8-10 m; 3 , Terraza a +3-5 m; 4, Llanura aluvial; 5, Cono aluvial; 6, Fondo de valle; 7, Depósitos coluviales; 8, Red de drenaje; 9 , Mapa base MNT25 del Instituto Geográfico Nacional.

Figure 2. Fluvial terraces in the Pinedo area, upper Arlanzón valley. Legend: 1 , terrace at $+12-15 m ; 2$, terrace at $+8-10 m ; 3$, terrace at $+3-5$ m; 4, floodplain; 5, alluvial cone; 6, valley bed; 7, colluvial deposits; 8, drainage network; 9, base map MNT25 of the National Geographical Institute. 
Benito-Calvo, A., et al., 2018. Evolución geodinámica pleistocena del valle del río Arlanzón... Boletín Geológico y Minero, 129 (1/2): $59-82$

Los datos cronológicos existentes y los saltos topográficos entre terrazas fluviales (estimados con el MDT05-LiDAR), fueron usados para estimar la variación de los ratios de incisión en la evolución del relieve. Los datos relativos a composición mineralógica por Difracción de Rayos $\mathrm{X}$ y fueron realizados en el Laboratorio de Arqueométrica del CENIEH.

\section{El valle del río Arlanzón}

El río Arlanzón surge de la ladera norte del Pico San Millán, (1685 m s.n.m., MNT25, IGN). En base a los trazados del valle, su arquitectura fluvial y a la pendiente de su curso, el valle del río Arlanzón ha sido subdividido en tres tramos (Fig. 1). A continuación se describen las secuencias fluviales en estos tres tramos, centrándose especialmente en su tramo medio, donde se localiza la Sierra de Atapuerca y sus conjuntos arqueológicos. Dentro del tramo medio se incluyen los valles de los ríos Vena y Pico, tributarios del río Arlanzón en esta zona, que drenan la parte central y norte de la Sierra de Atapuerca.

\section{Tramo alto: Pico de San Millán-Embalse de Úzquiza}

El valle alto del río Arlanzón presenta pendientes medias del $2.38 \%$, y fluye flanqueado por las estribaciones de San Millán y Trigaza (2132 m s.n.m.), al este, y la sierra de Mencilla (Pico Mencilla, 1932 m s.n.m.), al oeste. El valle principal presenta una dirección general SO-NE, encajado en materiales cámbricos y carboníferos fallados con esta misma dirección (Fig. 1). Esta dirección se mantiene hasta el Embalse del Arlanzón, donde el valle adquiere una dirección N-S.

En su tramo alto, el valle del río Arlanzón presenta escasos depósitos cuaternarios, a excepción de las manifestaciones periglaciares y los sistemas glaciares (García, 1979). Aguas arriba del Embalse del Arlanzón, en el fondo del valle se desarrollan conos procedentes de los torrentes laterales y terrazas bajas cuyos planos se sitúan a +3-5, +8-10 m y +12-15 m sobre el cauce (Fig. 2). Por otro lado, aguas abajo del Embalse del Arlanzón, se han podido diferenciar niveles de terraza fluviales actualmente inundadas por el Embalse de Úzquiza (Fig. 3), a cotas de $+30,+13-17$ y +8-9 m sobre el cauce (Fig. 3), que se continúan con los niveles situados aguas abajo de la Presa de Úzquiza. Por encima de estos niveles con depósitos fluviales, se observan numerosos planos de hombreras y rellanos erosivos que marcan niveles de base escasamente estudiados hasta la fecha (Benito-Calvo and Pérez-González, 2007; Arnáez, 1987; Lemartinel, 1985).

\section{Tramo medio: Embalse de Úzquiza-Villabilla de Burgos}

En este tramo el río discurre con pendientes medias del $0.47 \%$, y en un punto intermedio drena el relieve anticlinal de la Sierra de Atapuerca (Benito-Calvo and Pérez-González., 2015; Pérez-González et al., 2001; Pérez-González et al., 1999; Pineda and Arce, 1997; Olivé et al., 1990; Zazo et al., 1983, 1987; Figs. 1 y 4). En el entorno de la población de Arlanzón, el valle sale a los materiales neógenos (Mioceno inferiorsuperior) y se dispone con la orientación general característica de este tramo, casi E-O (Figs. 1 y 4). Esta orientación está formada por tres tramos largos de tendencia ENE-OSO, articulados por dos tramos menores de dirección NO-SE (Fig. 4). Dicho patrón debe tener su origen en respuesta a alineaciones estructurales del sustrato, al igual que los escalones o knickpoints observados en el perfil longitudinal del valle (Benito-Calvo et al., 2008), o la asimetría del valle. Entre Arlanzón y Burgos, esta asimetría se define por una morfología escalonada con buen desarrollo de terrazas bajas en la margen derecha (terrazas T10-T14), mientras que el cauce circula pegado a la margen izquierda del valle (Figs. 4 y $5 \mathrm{C}$ ), formando un escarpe fluvial con presencia frecuente de deslizamientos (Benito-Calvo and Pérez-González, 2015).

Los bordes del valle medio del río Arlanzón están constituidos por las superficies neógenas (BenitoCalvo and Pérez-González, 2007), que se alzan a +72$120 \mathrm{~m}$ m del río actual. En este tramo se encuentra la secuencia de terrazas mejor representada del valle del río Arlanzón (Benito-Calvo and Pérez-González, 2015; Benito, 2004, Pérez-González et al., 2001; Pineda and Arce, 1997; García et al., 1997; Zazo et al., 1983, 1987). Estas terrazas presentan espesores medios de 2-4 m, con sus bordes coluvionados $y$, a menudo, con conos aluviales laterales que cubren sus depósitos (Figs. 4 y 5 ).

El plano de terraza más antiguo, o T1, tiene su representación más clara al sur de Burgos (Fig. 4), (cerros Guerra, 935 m s.n.m., y de La Serna, 935 m s.n.m.), donde el borde del plano de la terraza se alza entre los $+90 y+100 \mathrm{~m}$ respecto al cauce del Arlanzón (Fig. 5E), a nivel de la superficie SE4-PI (páramo inferior) o encajados en ella 5-6 m. Por debajo, se encuentra la terraza T2 (+82-91 m) (Fig. 4), que también se localizada en el borde de la sierra de la Demanda, y al suroeste de la Sierra de Atapuerca (Fig. 5D). La terraza T3 $(+70-78 \mathrm{~m})$, forma grandes planos entre Arlanzón, y San Millán de Juarros, y al sur de Burgos (Figs. 4 y 5). En esta última localización, una gravera deja expuestos sus sedimentos (Fig. 6A), compuestos por barras de clastos de cuarcita granosoportados, 


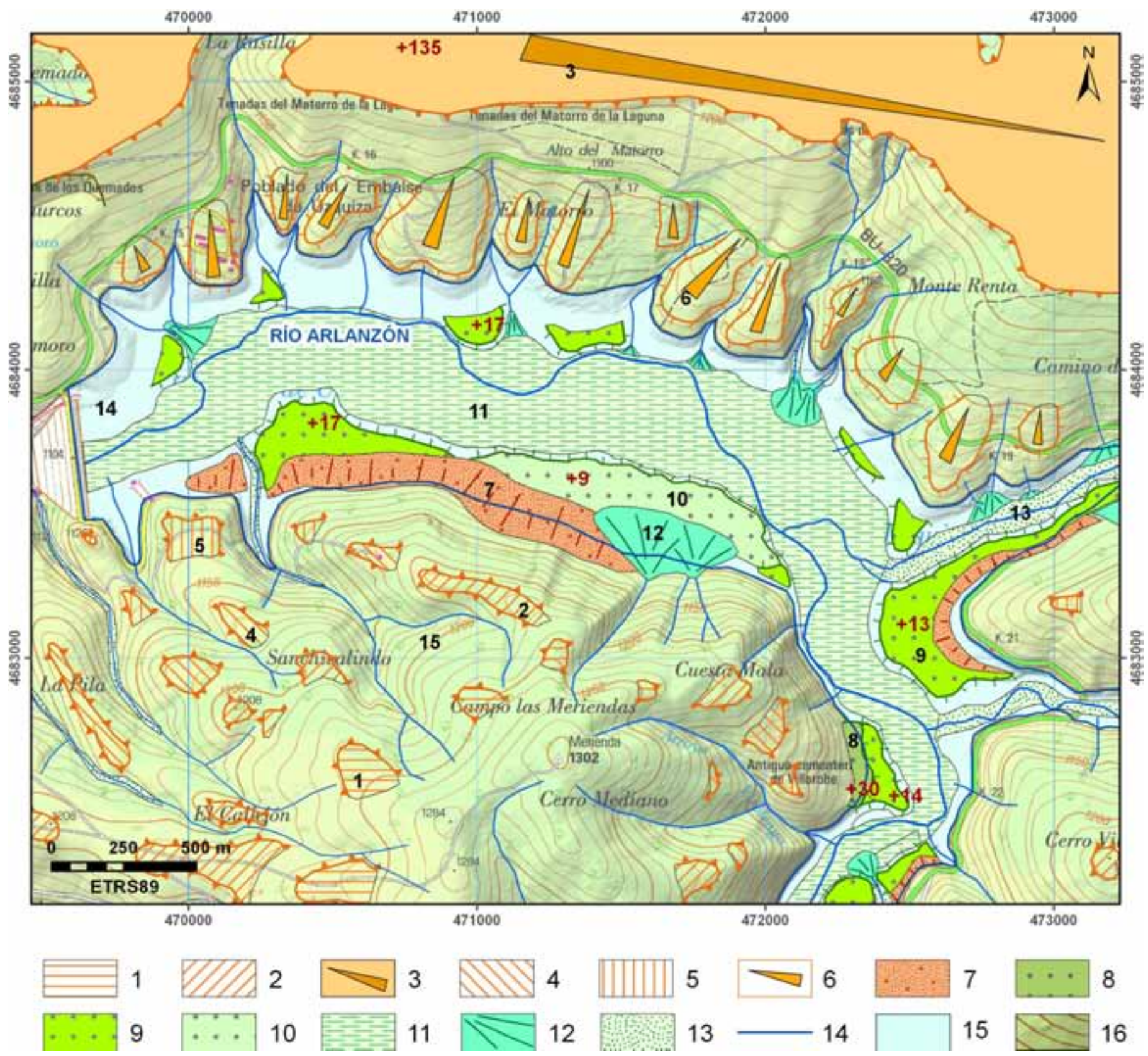

Figura 3. Terrazas fluviales en el Embalse de Úzquiza. Leyenda: 1, Superficie de erosión SE1; 2, Superficie de erosión SE2; 3, Nivel de piedemonte aluvial correlativo a SE2; 4, Superficie de erosión SE3; 5, Superficie de erosión SE4; 6, Glacis erosivos; 7, Depósitos coluviales; 8, Terraza a +30 m; 9; Terraza a +17-13 m; 10, Terraza a +8-9 m; 11, Llanura aluvial; 12, Cono aluvial; 13, Fondo de valle; 14, Red de drenaje; 15, Área indundada por el Embalse de Úzquiza; 16, Mapa base MNT25 del Instituto Geográfico Nacional.

Figure 3. Fluvial terraces in the Úzquiza Reservoir. Legend: 1, erosion surface SE1; 2, erosion surface SE2; 3, Piedmont level correlative to SE2; 4, erosion surface SE3; 5 , erosion surface SE4; 6 , erosive glacis; 7, colluvial deposits; 8, terrace at $+30 \mathrm{~m} ; 9$; terrace at $+17-13 \mathrm{~m}$; 10 , terrace at +8-9 m; 11, floodplain; 12, alluvial cone; 13, valley bed; 14, drainage network; 15, area flooded by the Úzquiza Reservoir; 16, base map MNT25 of the National Geographical Institute.

que incluyen un nivel intercalado de arenas masivas, cuya datación por ESR ha aportado una única edad de $1.14 \pm 0.13 \mathrm{Ma}$ (Moreno et al., 2012).

La terraza T4 (+60-67 m), presenta una distribución constante a lo largo de este tramo (Figs. 4 y $6 \mathrm{~B}$ ). En una gravera situada al este de Villacienzo (Figs. 4 y $5 \mathrm{E})$, se observaban más de $4 \mathrm{~m}$ de barras de gravas cuarcíticas, que intercalan bancos y canales de arenas gruesas y medias-finas, aparentemente masivas. En la mineralogía de los niveles arenosos, estimada por 


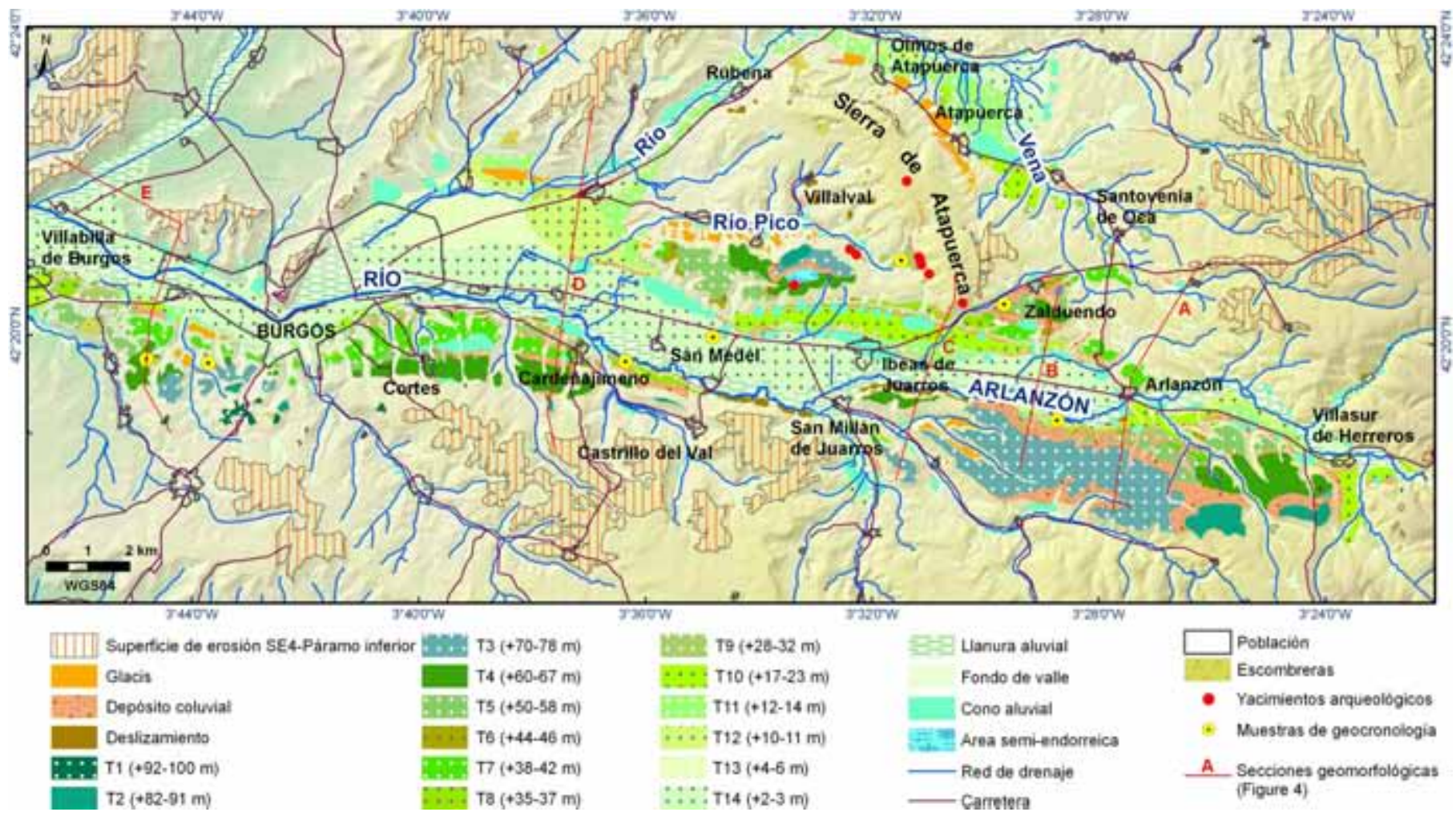

Figura 4. Depósitos cuaternarios en el valle medio del río Arlanzón.

Figure 4. Quaternary deposits in the middle Arlanzón valley.

DRX semicuantitativo, domina el cuarzo, seguido de lejos por filosilicatos, y en menor proporción plagiocasas, feldespato potásico y óxidos de Fe (Tabla 1). La datación de un nivel de arenas situado a 2-3 m del techo (Moreno, 2011), ha proporcionado dos edades ESR de $0.78 \pm 0.12 \mathrm{Ma}$ y $0.93 \pm 0.10 \mathrm{Ma}$ (Moreno et al., 2012). En esta última localización, datos preliminares de paleomagnetismo han proporcionado polaridades inversas para estos depósitos (Benito-Calvo et al., 2008).

Por debajo de T4, se encaja el nivel T5 que alcanza cotas relativas respecto al cauce de $+50-58 \mathrm{~m}$. Al oeste de Zalduendo, esta terraza presenta un espesor anómalo visto de $7.5 \mathrm{~m}$ donde se observan gravas cuarcíticas y niveles limo-arenosos, compuestos principalmente por cuarzo (Tabla 1). Los sedimentos de esta gravera presentan signos claros de deformación (Fig. 6C), representados por fracturas, embudos de hundimiento, inyecciones y pliegues. Estas deformaciones, más tenues hacia techo, parecen estar relacionadas con la plasticidad del sustrato, representado por arcillas y margas que también afloran deformadas en la gravera. En los niveles limo arenosos de esta gravera se realizaron tres dataciones por ESR (Figs. 4 y $5 \mathrm{E}$ ) que dieron edades de $0.70 \pm 0.10$;
$0.70 \pm 0.07$ y $0.60 \pm 0.11 \mathrm{Ma}$ (Moreno et al., 2012), mientras que medidas paleomagnéticas preliminares proporcionaron polaridades normales (Benito-Calvo et al., 2008).

En estas terrazas altas, Pérez-González et al. (1999), describen suelos Alfisols de tipo Palexeralf, con horizontes $\mathrm{A} / \mathrm{E} / \mathrm{Bt}$, que incluyen contenido homogéneo de arcillas de iluviación, películas de arcilla gruesa y procesos de alteración notables en los clastos de cuarcita.

Encajado en T5, se localizan las terrazas T6 $(+44-46$ m) y T7 (+38-42 m), cuya diferenciación es difícil en este tramo. Las terrazas situadas entre el nivel de T4 (+60-67 m) y T7 (+38-42 m), que se apoyan sobre las calizas astaracienses situadas al este de la Sierra de Atapuerca, aumentan localmente de cota relativa aguas abajo. Las facies micríticas con sílex de estas calizas astaracienses, parecen haber constituido un nivel resistente a la erosión que habría condicionado tasas de incisión menores, de carácter local.

A alturas relativas de $+35-37 \mathrm{~m}$ se desarrolla una terraza más reciente (T8) Los materiales de la terraza al este de Cardeñajimeno, están compuestos por $4 \mathrm{~m}$ de gravas con cantos imbricados y arenas limo-arcillosas (Fig. 6E). Del mismo modo que en niveles anteriores, la mineralogía de las facies finas está domina- 
Benito-Calvo, A., et al., 2018. Evolución geodinámica pleistocena del valle del río Arlanzón... Boletín Geológico y Minero, 129 (1/2): 59-82
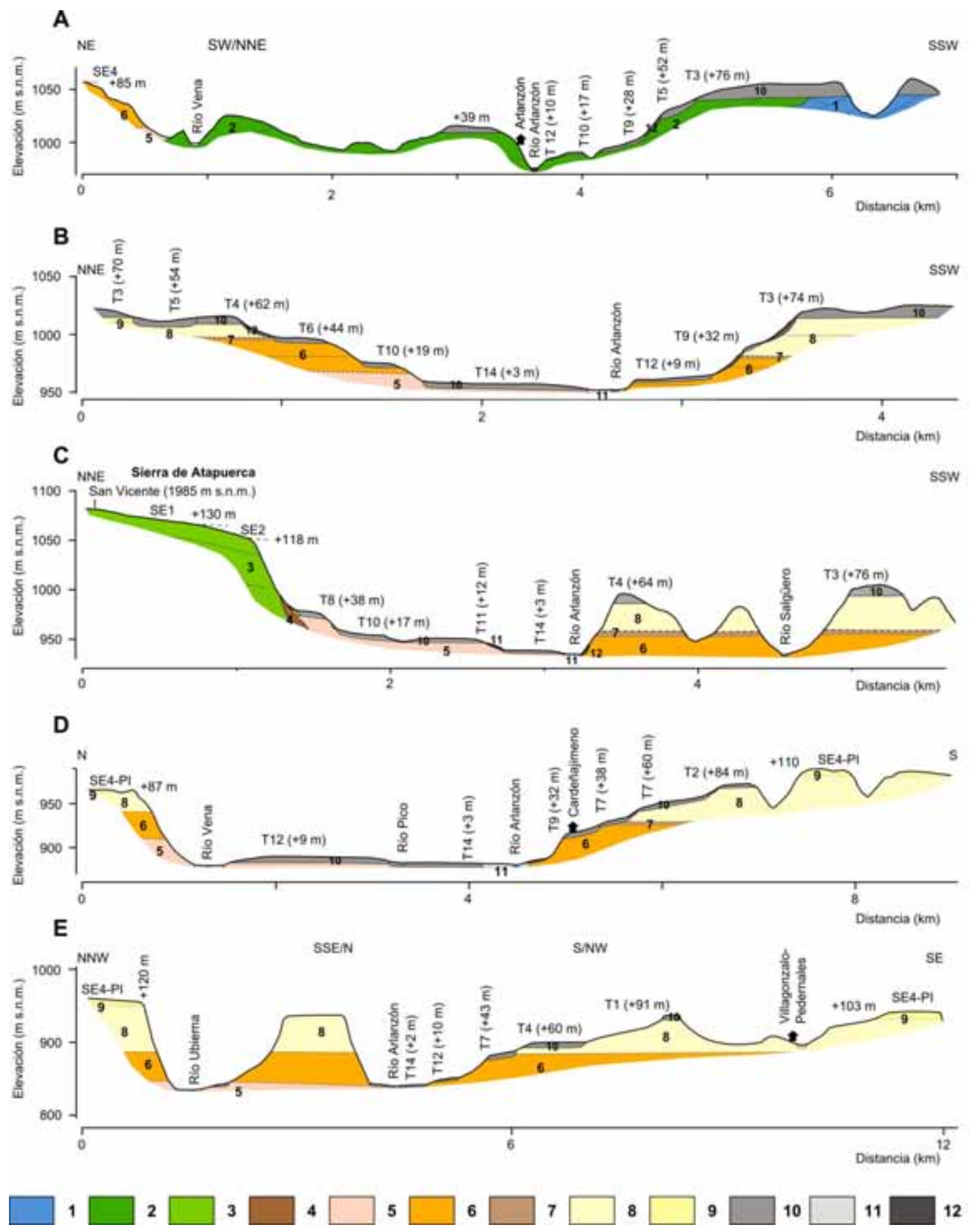

Figura 5. Cortes geomorfológicos del valle medio del río Arlanzón. Leyenda: 1, Carbonatos y margas (Jurásico); 2, Conglomerados, areniscas y calizas (Cretácico inferior); 3, Calizas y dolomías (Cretácico superior); 4, Conglomerados, arenas, limos y calizas (OligocenoMioceno inferior); 5, Margas, arcillas y evaporitas (Mioceno inferior); 6, Gravas, arenas y arcillas (Mioceno medio, Astaraciense); 7, Calizas (Mioceno medio, Astaraciense); 8, Margas, arcillas y calizas (Mioceno superior); 9, Calizas del páramo inferior (Mioceno superior); 10, Terraza fluvial (Pleistoceno-Holoceno); 11, Llanura aluvial y fondos de valles; 12, Depósitos coluviales y deslizamientos.

Figure 5. Geomorphological cross-sections of the middle Arlanzón valley. Legend: 1, carbonates and marls (Jurassic); 2, conglomerates, sandstones and limestones (Early Cretaceous); 3, limestones and dolostones (Upper Cretaceous); 4, conglomerates, sands, silts and clays (Oligocene-Early Miocene); 5, marls, clays, and evaporites (Early Miocene); 6, gravels, sands and clays (Middle Miocene, Astaracian); 7, limestones (Middle Miocene, Astaracian); 8, marls, clays and limestones (Late Miocene); 9, Lower páramo limestones (Late Miocene); 10, fluvial terraces (Pleistocene-Holocene); 11, floodplain and valley beds (Holocene); 12, colluvial deposits and landslides. 
Benito-Calvo, A., et al., 2018. Evolución geodinámica pleistocena del valle del río Arlanzón... Boletín Geológico y Minero, 129 (1/2): $59-82$

\begin{tabular}{|l|c|c|c|c|c|c|}
\hline Nivel / \% & Cuarzo & Calcita & Feldespato K & Plagioclasas & Filosilicatos & Óxidos de Fe \\
\hline Terraza T8 (+35-37 m) & 81 & 0 & 3 & 5 & 8 & 3 \\
\hline Terraza T5 (+50-58 m) & 79 & 0 & 4 & 5 & 10 & 2 \\
\hline Terraza T4 (+60-67 m) & 72 & 0 & 5 & 6 & 13 & 4 \\
\hline
\end{tabular}

Tabla 1. Composición mineralógica de las facies finas de las terrazas altas y medias del valle del río Arlanzón.

Table 1. Mineralogical composition of the fine fraction of the high and middle terraces of the middle Arlanzón valley.

da por el cuarzo (Tabla 1), que aumenta su proporción respecto a terrazas más antiguas. Este aumento en cuarzo está en contraposición al descenso en los filosilicatos (incluyendo los minerales de arcillas), más abundantes en las terrazas más antiguas. Dos dataciones ESR realizadas en este afloramiento (Figs. 4 y $5 \mathrm{E})$, han proporcionado edades de $0.37 \pm 0.07 \mathrm{y}$ $0.40 \pm 0.09 \mathrm{Ma}$ (Moreno et al., 2012). El nivel T8 es difícilmente distinguible de la terraza que se desarrolla por debajo, o T9, la cual adquiere elevaciones relativas que oscilan entre $+28 \mathrm{~m}$ al sur de Arlanzón y los +28-32 $\mathrm{m}$ que adquiere al Sur de Burgos, donde los términos gravosos incluyen fragmentos de sílex erosionados del Neógeno.

La terraza fluvial que se encaja a continuación, T10, tiene una representación considerable (Fig. 4). Por debajo, se emplazan las terrazas bajas del río Arlanzón T11 (+12-14 m),T12 (+8-11 m), T13 (+4-6 m), y $\mathrm{T} 14(+2-3 \mathrm{~m})$, especialmente conservadas en la zona de confluencia de los ríos Arlanzón y Vena (Figs. 4 y 5D). Pérez-González et al. (1999), definen los suelos de las terrazas bajas como Alfisols Haploxeralfs, con perfiles tipo A/Bt.

El nivel de T11 se lo puede observar en la margen izquierda y aguas abajo de Arlanzón, apoyada sobre la facies Santa María del Campo, donde se distinguen gravas cuarcíticas con 2 metros intercalados de arenas finas limosas, que han proporcionado una edad ESR de $0.14 \pm 0.07 \mathrm{Ma}$ (Moreno et al., 2012). La terraza más reciente diferenciada, T14 $(+2-3 \mathrm{~m})$, se desarrolla a lo largo de todo el tramo (Figs. 4 y 5), donde localmente aún conserva los trazos de canales abandonados. Esta terraza fue datada por TL en el Laboratorio de Datación y Radioquímica de la Universidad Autónoma de Madrid (Benito-Calvo et al., 2008), obteniendo una edad de $4.827 \pm 338$ BP para un nivel de arenas muestreado en una gravera actualmente colmatada que se sitúa al este de San Medel (Fig. 4).

Por último, la llanura aluvial del río Arlanzón presenta anchuras de $250-700 \mathrm{~m}$ y alturas relativas respecto al canal de $+0-1 \mathrm{~m}$. En las zonas con menos modificaciones antrópicas, la llanura presenta un denso entrelazado de canales secundarios (BenitoCalvo and Pérez-González, 2015), además de barras fluviales activas. En estos sedimentos se desarrollan suelos Fluvent con perfiles $A / C$, tonos pardos (10YR) y ausencia de películas de arcilla (Pérez-González et al., 1999).

\section{Valle del río Vena}

El curso del río Vena describe un arco convexo hacia el norte, que transcurre entre su nacimiento en la vertiente occidental de los Montes de Oca (a $1193 \mathrm{~m}$ s.n.m.), y su afluencia al río Arlanzón (Fig. 4), en la ciudad de Burgos (a 853 m s.n.m.). Durante este trazado el río Vena drena los materiales cretácicos, jurásicos y oligocenos del flanco este y el extremo norte de la Sierra de Atapuerca, así como materiales neógenos del Mioceno inferior, medio y superior (facies Dueñas, Villatoro, Pedraja, Cuestas y calizas del páramo inferior) (Fig. 1). El valle del río Vena está delimitado por las superficies de erosión neógenas Pedraja (Benito-Calvo and Pérez-González, 2007), y por plataformas estructurales preservadas en calizas miocenas.

Las formas más antiguas del valle del río Vena se conservan en la zona de cabecera, formadas por las terrazas altas compartidas con el río Arlanzón T2-T7, y planos erosivos equiparables, localizados sobre la facies Pedraja. En las laderas de la Sierra de Atapuerca (zona de Olmos de Atapuerca), también se reconocen terrazas rocosas labradas sobre los materiales mesozoicos a +50 (T5) $,+66(\mathrm{~T} 4),+70$ (T3) y +80 $\mathrm{m}$ (T2). En el sector de Zalduendo-Arlanzón, el valle del río presenta niveles de terraza a +15-32, +6-36, +4$23,+1-14,+1-11, y+1-4 \mathrm{~m}$ respecto al cauce. La distribución longitudinal de estos niveles de terraza sugiere una geometría divergente aguas abajo (Benito, 2004), hasta adquirir cotas relativas propias del río Arlanzón en la confluencia de ambos río en Burgos.

Los depósitos observados en las terrazas del río Vena, están formados por una capa superficial de clastos redondeados, entre las que dominan las cuarcitas frente a pizarras y metareniscas, todas ellas procedentes de la erosión de la facies Pedraja, cuya área fuente se circunscribe al Paleozoico de la Demanda. 


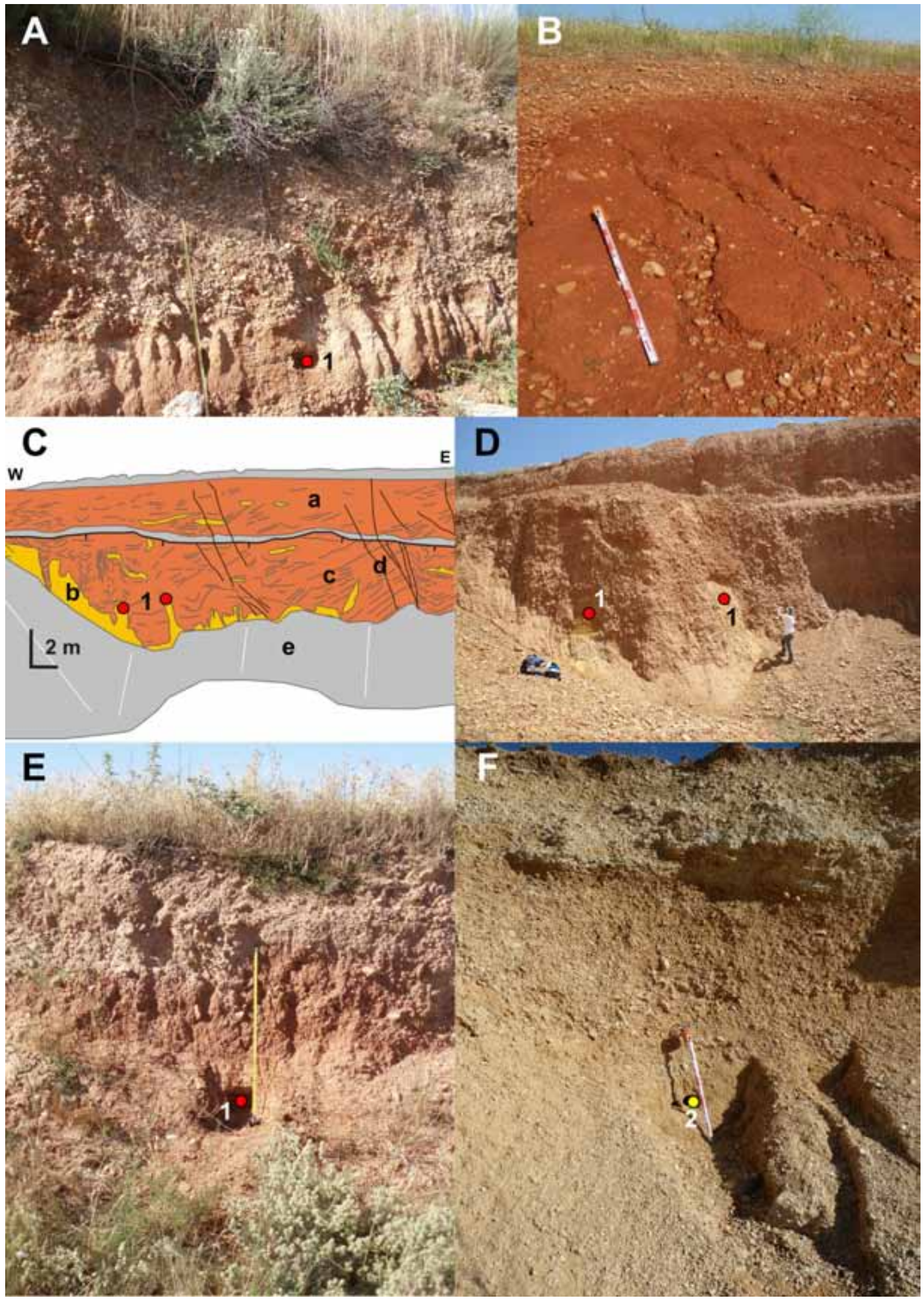


Benito-Calvo, A., et al., 2018. Evolución geodinámica pleistocena del valle del río Arlanzón... Boletín Geológico y Minero, 129 (1/2): $59-82$

\section{Valle del río Pico}

El valle alto del río Pico, denominado valle de La Hoyada, incide los carbonatos del Cretácico superior y los conglomerados oligocenos de la Sierra de Atapuerca. A su salida de la Sierra de Atapuerca, el valle de la Hoyada drena los materiales Neógenos, donde recibe aguas del valle del Valhondo. Aguas abajo de la confluencia Hoyada-Valhondo, el río Pico discurre en dirección oeste a través de un valle estrecho, encajado por la margen izquierda en las terrazas medias y altas del río Arlanzón (T2-T7, Fig. 4) y por la margen derecha en el plano de Villalval-Rubena (Benito-Calvo and Pérez-González, 2015; Benito, 2004), que se conserva sobre el techo calizo de las facies del mioceno medio (Astaraciense). Actualmente, el tramo bajo del río Pico se encuentra drenado (Fig. 4), desaguando en el río Arlanzón a su entrada en Burgos.

En la confluencia Hoyada-Valhondo, el valle del río Pico preserva una terraza fluvial colgada a $+6-10 \mathrm{~m}$ (Fig. 4), formada por $4 \mathrm{~m}$ vistos de gravas subangulosas de calizas cretácicas con cementaciones locales, que incluyen niveles arenosos y areno-arcillosos, más abundantes hacia la base (Fig. 6F). Esta terraza ha sido correlacionada con el nivel T9 $(+28-32 \mathrm{~m})$ del tramo medio del río Arlanzón (Benito, 2004), y ha sido datada mediante OSL y TT-OSL en torno a los $348 \pm 16$ $\mathrm{Ka}$ (Arnold et al., 2013). Estas edades son ligeramente más jovenes que las edades obtenidas por ESR para T8, de $0.37 \pm 0,07$ y $0.40 \pm 0.09 \mathrm{Ma}$ (Moreno et al., 2012).

Aguas abajo, el valle es marcadamente asimétrico, definido por una margen derecha sin depósitos pleistocenos, donde confluyen varios arroyos laterales, y una margen izquierda donde se desarrollan glacis de ladera (Benito-Calvo and Pérez-González, 2015; Fig. 4), con pendientes del 3.5-7\%. Estos glacis forman hasta tres niveles $(a+4-6,+4-15,+8-24 \mathrm{~m}$ sobre el cauce del río Pico), de características erosivas o bien compuestos por una cobertera superficial de cantos redondeados cuarcíticos, y clastos subangulosos de caliza y sílex. Esta composición indica una procedencia de las terrazas del río Arlanzón, incluyendo mate- rial erosionado de la capa calcárea con nódulos de sílex del Astaraciense. El desmantelamiento de estos depósitos de glacis, genera en ocasiones depósitos coluvionares al pie del escarpe de los glacis, donde se conservan yacimientos del Paleolítico medio datados aproximadamente entre 71 y $48 \mathrm{Ka}$ (OSL single grain, Arnold et al., 2013).

\section{Tramo bajo y desembocadura: Villabilla de Burgos- Palenzuela}

Desde la población de Villalbilla de Burgos y coincidiendo con la confluencia del río Ubierna, el valle del río Arlanzón sufre una inflexión brusca hacia el SO, que se prolonga en líneas generales hasta la unión del río Arlanzón con el río Arlanza (Fig. 1). Este trazado de dirección NE-SO ha sido frecuentemente explicado por un accidente del zócalo tectónico (Pineda and Arce, 1997; Pérez-González et al., 1994).

En este tramo, el río Arlanzón presenta una pendiente media del $0.13 \%$, encajado del orden de +150 $\mathrm{m}$ en la superficie del páramo inferior. Durante este trazado el valle presenta una geometría asimétrica, más marcada aguas debajo de Buniel, donde la margen izquierda es escarpada, sin terrazas medias y altas, y con frecuentes conos aluviales (Benito, 2004; Pavón et al., 1973). Al contrario, la margen derecha presenta una geometría más escalonada por la presencia de afloramientos de terraza (Pavón et al., 1973), que han sido medidos a +9-10, +22-26, +41-42, +50 y $+66 \mathrm{~m}$ sobre el cauce.

El desarrollo más completo de la secuencia de terrazas de este tramo (Fig. 7), se localiza en la transversal situada a la altura de Villodrigo (Núñez et al., 1975), donde el término superior de la secuencia, corresponde a un nivel de poca presencia, que se alza $a+62-59 \mathrm{~m}$ (T4) respecto al cauce y que se encaja en un plano estructural (Benito, 2004). A partir de este nivel, se desarrollan terrazas a $+53 \mathrm{~m}(\mathrm{T5}),+45-46 \mathrm{~m}$ (T6), +40 m (T7), +32 m (T9), +19-20 m (T10), +13 m (T11), +10-11 m (T12), +4-6 m (T13) y +2-3 m (T14). EI nivel más bajo, está colgado respecto al cauce, como se puede observar al oeste de Villodrigo, donde el río

Figura 6. Depósitos de terraza en el valle medio del río Arlanzón. A) Terraza T3 (+70-78 m), localizada al sur de Burgos. B) TerrazaT4 (+60$67 \mathrm{~m}$ ) situada en Cortes. C) Rasgos de deformación en los depósitos de la terraza T5 (+50-58 m), situada en Zalduendo. Leyenda: a, gravas; b, limos arenosos; c, planos de estratificación; d, fracturas; e, coluvión. D) Terraza T5 (+50-58 m), localizada en Zalduendo. E) Terraza T8 (+35-37 m), situada al oeste de Cardeñajimeno. F) Terraza a +6-10 m, localizada en el valle alto del río Pico. Puntos de muestreo: 1 , ESR; 2, TT-OSL.

Figure 6. Terrace deposits in the middle Arlanzón valley. A) Terrace T3 (+70-78 m), toward the south of Burgos. B) Terrace T4 (+60-67 m) in Cortes. C) Deformation features in terrace T5 $(+50-58 \mathrm{~m})$, located in Zalduendo. Legend: a, gravels; $b$, sandy silts; $c$, deformed bedding; $d$, fractures; e, colluvium. D) Terrace T5 $(+50-58 \mathrm{~m})$ in Zalduendo. E) Terrace T8 $(+35-37 \mathrm{~m})$ in Cardeñajimeno. F) Terrace at $+6-10 \mathrm{~m}$, located in the upper Pico valley. Sampling: 1, ESR; 2, TT-OSL. 


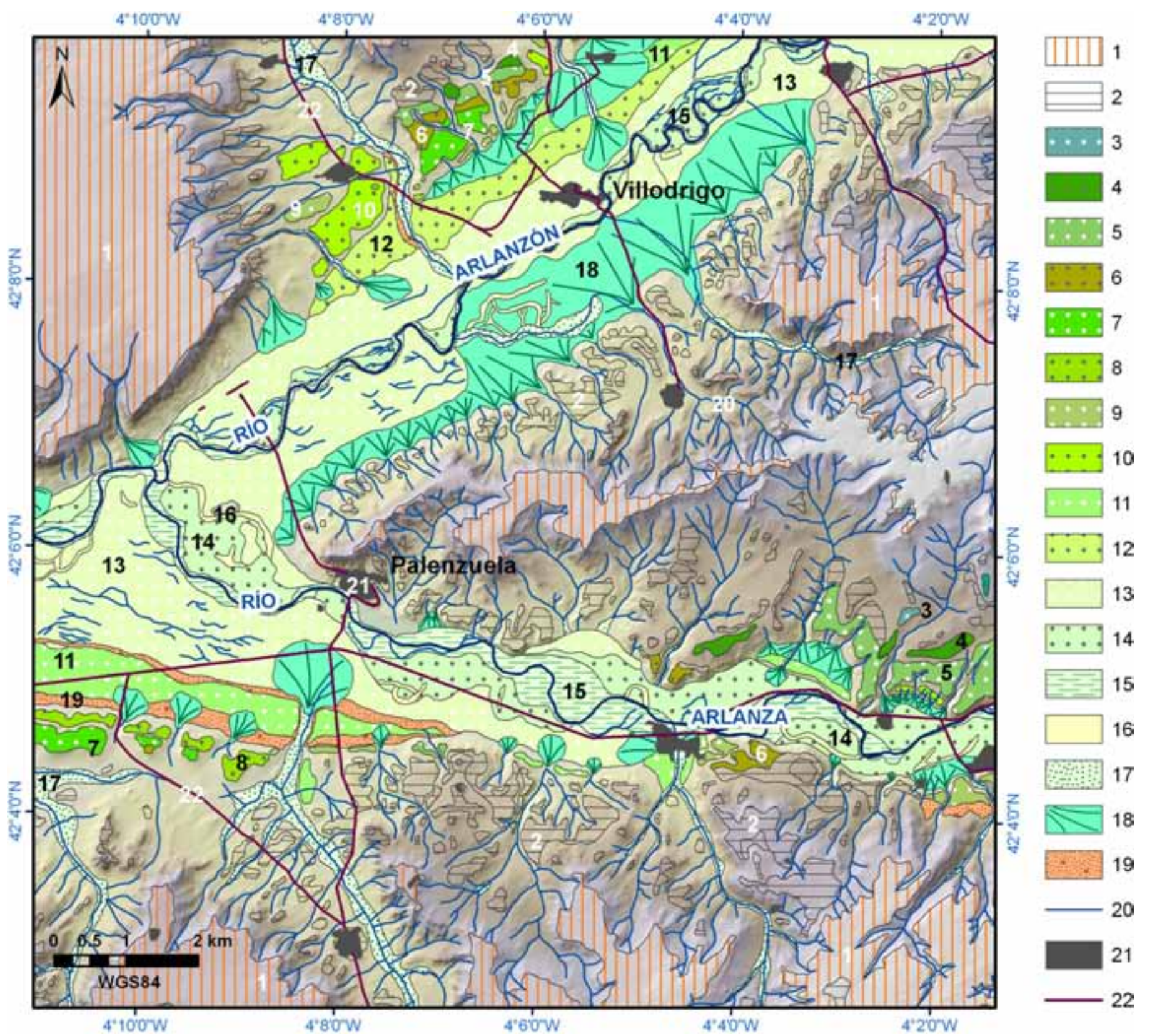

Figura 7. Depósitos cuaternarios en el tramo bajo del valle del río Arlanzón. Leyenda: 1, Superficie de erosión SE1-páramo inferior; 2 , Plataformas estructurales; 3, Terraza T3 $(+70-78 \mathrm{~m})$; 4, Terraza T4 (+60-67 m); 5, Terraza T5 (+53 m); 6, Terraza T6 (+45-46 m); 7, TerrazaT7 (+40 $\mathrm{m}) ; 8$, Terraza T8 (+36 m); 9, Terraza T9 (+32 m); 10, Terraza T10 (+19-20 m); 11, TerrazaT11 (+13 m); 12, Terraza T12 (+10-12 m); 13, TerrazaT13 (4-6 m); 14, TerrazaT14 (+2-3 m); 15, Llanura aluvial; 16, Canales abandonados; 17, Fondos de valle, 18, Conos aluviales; 19, Depósitos coluviales; 20, Drenaje; 21, Poblaciones; 22, Carreteras.

Figure 7. Quaternary deposits in the lower Arlanzón valley. Legend: 1, erosion surface SE1-lower páramo; 2, structural platform; 3, terrace T3 $(+70-78 \mathrm{~m}) ; 4$, terrace T4 $(+60-67 \mathrm{~m}) ; 5$, terrace T5 $(+53 \mathrm{~m}) ; 6$, terrace T6 $(+45-46 \mathrm{~m}) ; 7$, terrace T7 $(+40 \mathrm{~m}) ; 8$, terrace T8 $(+36 \mathrm{~m}) ; 9$, terrace T9 (+32 m); 10, terrace T10 $(+19-20 \mathrm{~m}) ; 11$, terraceT11 $(+13 \mathrm{~m}) ; 12$, terrace T12 $(+10-12 \mathrm{~m}) ; 13$, terrace T13 $(4-6 \mathrm{~m}) ; 14$, terrace T14 $(+2-3 \mathrm{~m}) ; 15$, floodplain; 16, abandoned channel; 17, valley bed; 18, alluvial cone; 19, colluvial deposits; 20, drainage network; 21, population; 22, road.

incide en las margas del Mioceno inferior. Dichos niveles de terraza se distribuyen hasta la zona de confluencia, donde el río Arlanza desarrolla aguas arriba los mismos términos, incluyendo términos más antiguos (Fig. 7).

\section{Evolución geodinámica}

El valle del río Arlanzón empieza a labrarse a partir de una superficie de erosión (SE4-PI, Benito-Calvo and Pérez-González, 2007). A partir de esta superficie, el 
Benito-Calvo, A., et al., 2018. Evolución geodinámica pleistocena del valle del río Arlanzón... Boletín Geológico y Minero, 129 (1/2): $59-82$

valle se encaja controlado por estructuras del sustrato de direcciones E-O, NE-SO, NO-SE $y$, en menor medida, N-S. Estos trazados rectilíneos han sido frecuentemente explicados como accidentes tectónicos (Benito, 2004; Zazo et al., 1983, Pineda and Arce, 1997; Pérez-González et al., 1994). Dentro de estos trazados, el valle del río Arlanzón tiende a generar geometrías asimétricas, con márgenes derechas más escalonadas, y márgenes izquierdas más escarpadas, que indican la migración del valle y podrían estar debidas a posibles ajustes de los bloques del sustrato delimitados por dichos accidentes tectónicos.

El valle del río Arlanzón muestra un sistema escalonado de terrazas fluviales, sintetizado en la Fig. 8. Los niveles más antiguos (T1-T3), se hallan ligeramente encajados o a nivel de la superficie SE4/PI, especialmente en las zonas de enlace con los relieves alpinos. Por otro lado, el río actual circula incidido en las terrazas bajas y, en ocasiones, erosionando al sustrato mioceno. Esta circunstancia y el escaso desarrollo de la llanura aluvial, sugiere un predominio actual de los fenómenos de incisión vertical, frente a la sedimentación y a la expansión lateral.

Las terrazas fluviales se desarrollan en sistemas escalonados y principalmente colgados, formados principalmente por facies de canales de gravas aluviales. Los depósitos de gravas son mayoritariamente cuarcíticos, mientras que los niveles de finos muestran un predominio neto del cuarzo. El contenido en cuarzo aumenta en las terrazas inferiores (Tabla 1), mientras que las terrazas superiores contienen mayor proporción de filosilicatos, posiblemente relacionado con la mayor proporción de minerales de arcilla en las terrazas antiguas, a causa de la mayor duración de los procesos de iluviación.

En el valle del río Arlanzón, los sistemas de terrazas muestran un perfil longitudinal paralelo o subparalelo con variaciones locales relacionadas con la presencia de niveles más resistentes del sustrato mioceno (Benito-Calvo et al., 2008). Por otro lado, los valles menores del los ríos Vena y Pico, presentan terrazas con perfiles longitudinales divergentes. Tales geometrías divergentes indicarían tasas de incisión menores hacia la cabecera de dichos valles secundarios.

Las edades numéricas disponibles hasta el momento (Moreno et al., 2012), indican que las terrazas T3 $(+70-78 \mathrm{~m})$ y T4 $(+60-67 \mathrm{~m})$ se habrían formado en el Pleistoceno inferior (Fig. 8), con una edad aproximada de 1.14 Ma paraT3, y una edad media aritmética en torno a 0.85 Ma para T4. Esta terraza, además, presenta polaridades inversas atribuibles el Chron Matuyama (Benito-Calvo et al., 2008). El tránsito Pleistoceno inferior-medio ocurriría entre T4 (+60-67 m) y T5 (+50-58 m), ya que esta última terraza muestra edades medias de $0.66 \mathrm{Ma}$ y polaridades preliminares normales (Fig. 8). Durante el Pleistoceno medio, la terraza T9 $(+35-37 \mathrm{~m})$ se sitúa en edades medias de $0.385 \mathrm{Ma}$ y la terraza T8 $(+28-32 \mathrm{~m})$ se estima sobre los $0.348 \mathrm{Ma}$ (Fig. 8). Al final del Pleistoceno medio, o en el límite con el Pleistoceno superior, parece que se sitúa la terraza T11 $(+12-14 \mathrm{~m})$, con una edad alrededor de 0.14 Ma (Moreno et al., 2012). Esta edad situaría a las terrazas inferiores T12 y T13 en el Pleistoceno superior, mientras que el nivel de terraza T14 (+2-3 $\mathrm{m})$ proporcionó a priori una cronología holocena (Benito-Calvo et al., 2008; Fig. 8).

Esta secuencia cronológica es de difícil comparación con las edades mínimas de los planos erosivos datados por isótopos cosmogénicos en los Arribes del Duero (Antón et al., 2012), mientras que presenta muchas similitudes con las alturas relativas y cronologías disponibles en los sistemas fluviales escalonados de la depresión del Tajo (López-Recio et al., 2015; Pérez-González et al., 2013; Silva et al., 2013; Panera et al., 2011; Karampaglidis et al., 2011; Ortiz et al., 2009; Benito et al., 1998).

Las edades medias de las terrazas del valle del río Arlanzón y el tipo de depósitos que presentan sugieren, en principio, una correlación entre el desarrollo de las terrazas y los estadios isotópicos fríos, al igual que se ha observado en otros sistemas escalonados fluviales (Bridgland and Westaway, 2008; PérezGonzález et al., 2008). Considerando las curvas isotópicas publicadas por Lisiecki and Raymo (2005), el nivelT11 (+12-14 $\mathrm{m})$ del río Arlanzón se sitúa en el MIS 6. T9 $(+35-37 \mathrm{~m})$ y T8 $(+28-32 \mathrm{~m})$ coinciden en conjunto con el MIS 10. T5 $(+50-58 \mathrm{~m})$ se desarrolla en el MIS 16. T3 $(+70-78 \mathrm{~m})$ está en el entorno del MIS 34 . La terraza T4 (+60-67 m), quizás podría relacionarse con el MIS 22, aunque la edad media aritmética la sitúa en el MIS 21. Esta correlación preliminar indicaría que la agradación de las terrazas se realizaría principalmente asociada a los episodios fríos (Fig. 8), caracterizados por una mayor aridez que podría suponer un mayor aporte de sedimentos a los valles y un aumento de la carga de fondo en los cauces que produciría la agradación.

Son varios los trabajos que utilizan el salto entre terrazas y su cronología para calcular tasas de incisión fluvial, que en muchos casos asocian con el levantamiento tectónico regional (e.g. Bridgland and Westaway, 2008; Cunha et al., 2008). En estos trabajos, los cambios en cota del nivel de base fluvial se asocian con el levantamiento tectónico; en contraposición a los sistemas subsidentes, que generan secuencias apiladas, o los sistemas sin movimientos verticales, donde los cursos fluviales no se encajarían 


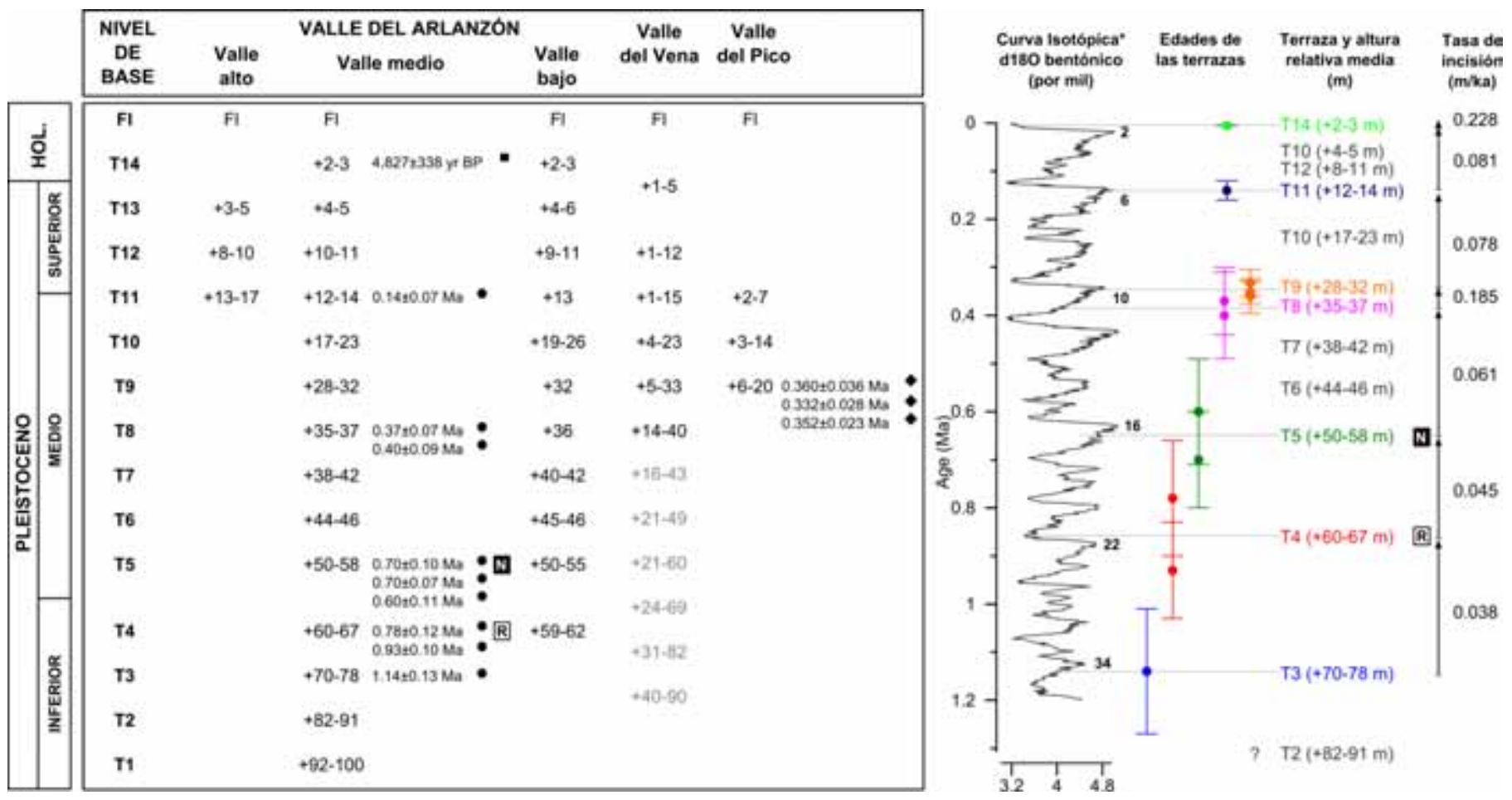

Figura 8. Cuadro de correlación, edades y tasas de incisión, de los niveles de base cuaternarios del valle del río Arlanzón, y de los valles menores del río Pico y el Vena, que drenan en conjunto la Sierra de Atapuerca. +91-100, alturas relativas de las terrazas en $\mathrm{m}$ respecto al cauce. Valores en gris, correspondientes a niveles erosivos. Círculos: edades ESR (Moreno et al., 2012); Rombos: edades TT-OSL (Arnold et al., 2013), Cuadrados: edades TL (Benito-Calvo et al., 2008). Medidas magnetoestratigráficas: N, polaridad normal; R, polaridad inversa. Curva isotópica: Lisiecki and Raymo (2005). Modificado de Benito-Calvo et al., (2017).

Figure 8. Correlation chart of the Arlanzón Quaternary base levels, including ages, erosion rates, and the Quaternary levels of the Vena and Pico valleys, which drained the northern and central part of the Sierra de Atapuerca. $+91-100$, terrace relative heights in $m$ above the channel. Grey values: erosive levels. Circle shape: ESR dates (Moreno et al., 2012); Diamond shape: TT-OSL dates (Arnold et al., 2013); Square shape: TL dates (Benito-Calvo et al., 2008). Magnetostratigraphic measures: $N$, normal polarity; $R$, reversed polarity (Benito-Calvo et al., 2008). *Isotopic curve: Lisiecki and Raymo (2005).

y fluirían en la misma posición a lo largo del tiempo (Bridgland and Westaway, 2008). De este modo, las tasas de incisión/levantamiento se usan para analizar la tectónica regional. Las edades descritas en las terrazas fluviales del río Arlanzón y su posición relativa respecto al cauce actual sugieren una ratio de incisión general, $y$ en posiciones centrales del valle (zonas de máxima incisión), de $0.064 \mathrm{~m} / \mathrm{ka}$, para el periodo comprendido desde el Pleistoceno inferior a la actualidad (T3-canal actual). Sin embargo, estas tasas han sido muy variables a lo largo del Cuaternario (Fig. 8). Así, para el tramo final del Pleistoceno inferior se han calculado tasas de 0.038 $\mathrm{m} / \mathrm{ka}$ (T3-T4); y de $0.045 \mathrm{~m} / \mathrm{ka}$ para el tránsito entre el Pleistoceno inferior y medio (T4-T5, Benito-Calvo et al., 2017). A lo largo del Pleistoceno medio, las tasas van incrementándose. Entre T8 y T9 se produce un pico con valores de $0.185 \mathrm{~m} / \mathrm{ka}$; mientras que entreT9 y T11 la tasa de incisión es más suave, estimándose en $0.078 \mathrm{~m} / \mathrm{ka}$ (Benito-Calvo et al., 2017). En el
Pleistoceno superior la tasa de incisión media continúa incrementándose paulatinamente, con valores de $0.081 \mathrm{~m} / \mathrm{ka}$. En el Holoceno, la tasa de incisión se dispara, ya que entre T14 y la llanura aluvial se obtienen los valores máximos, cifrados en $0.228 \mathrm{~m} / \mathrm{ka}$ (Fig. 8). Esto podría indicar un incremento brusco en la tendencia o que la edad de la terraza T14 (+2-3 m) está subestimada. A este respecto, conviene señalar que la terrazaT14 (+2-3 m), muestran perfiles edáficos que denotan cierta evolución (Pérez-González et al., 1999), con horizontes Bt desarrollados y continuos de colores pardo rojizos (5YR), que bien podrían retrotraerse al final del Pleistoceno.

De este modo, la tendencia conjunta muestra una aceleración de los ratios de incisión. Esta tendencia también se ha observado en otros muchos sistemas fluviales, fenómeno que es explicado por varios autores como una aceleración de las tasas de levantamiento tectónico (Bridgland and Westaway, 2008), variable en función de cada región. 


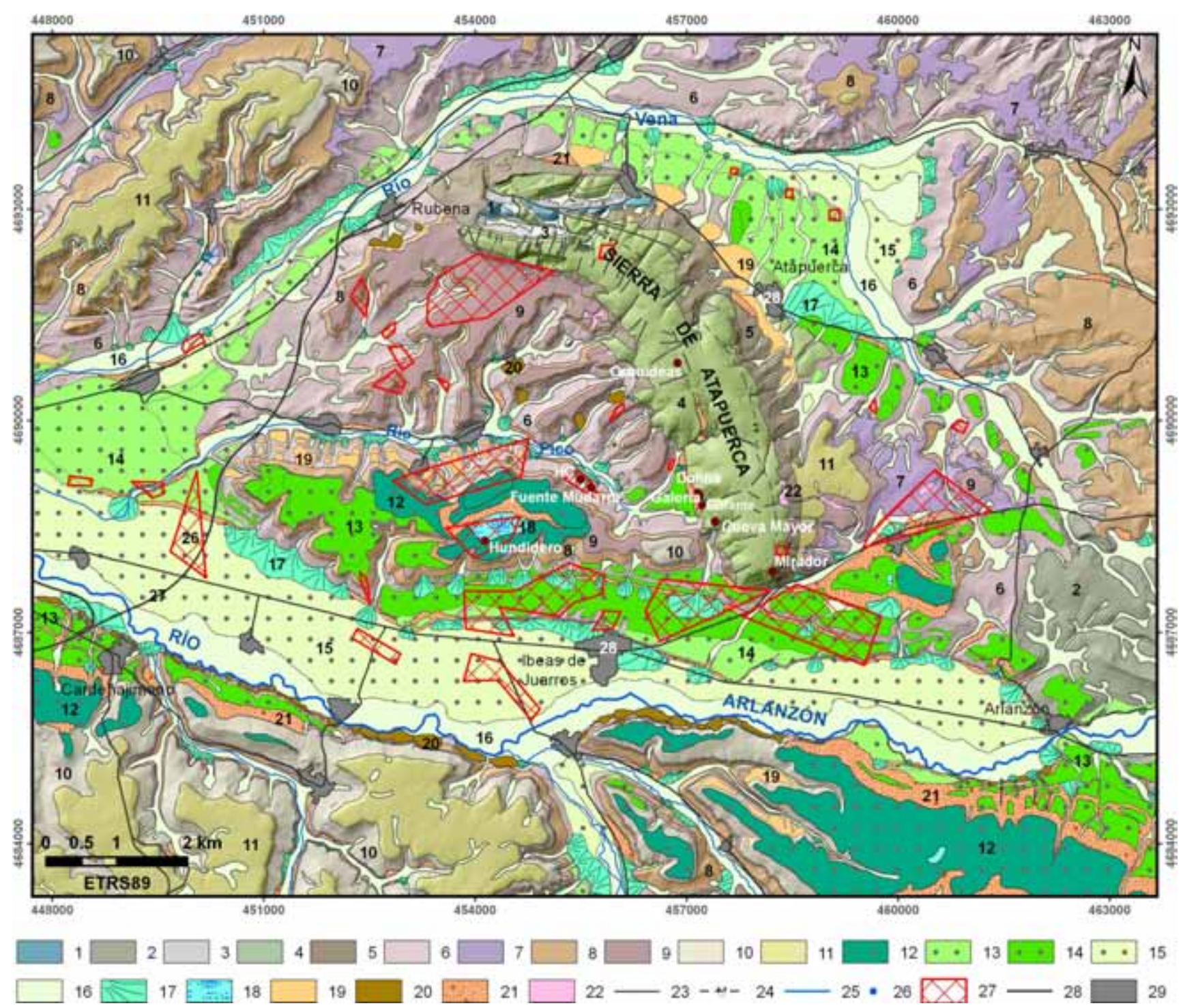

Figura 9. Distribución de los yacimientos arqueológicos de superficie sobre las formaciones geológicas y geomorfológicas. Cartografía geológica y geomorfológica basada en Benito-Calvo and Pérez-González (2015); distribución del material arqueológico basada en Navazo (2006). Leyenda: 1, Carbonatos (Jurásico); 2, Calizas, conglomerados y areniscas (facies Weald, Cretácico inferior); 3, Arenas, gravas y arciIlas (facies Utrillas, Cretácico inferior); 4, Carbonatos con nódulos locales de sílex (Cretácico superior); 5, Conglomerados, arenas, limos y arcillas (Oligoceno-Mioceno); 6, Margas, evaporitas y arcillas (facies Dueñas y Villatoro, Mioceno inferior); 7, Calizas (Mioceno inferior); 8, Conglomerados, arenas y arcillas (facies Pedraja, Tierra de Campos, Santa María del Campo, Mioceno medio, Astaraciense); 9, Calizas con nódulos métricos de sílex (Mioceno medio, Astaraciense); 10, Margas, calizas y arcillas (facies Dueñas, Mioceno superior); 11, Calizas del páramo inferior (Mioceno superior); 12, Terrazas fluviales del Pleistoceno inferior; 13, Terrazas fluviales del Pleistoceno medio; 14; Terrazas fluviales del Pleistoceno superior; 15, Terrazas fluviales del Pleistoceno superior-Holoceno; 16, Llanura aluvial y fondos de valle; 17, Conos aluviales; 18, Áreas semi-endorreicas y lagunas estacionales; 19, Glacis aluviales-coluviales; 20, Deslizamientos; 21, Depósitos coluviales; 22, Dolinas; 23, Fallas; 24, Anticlinal; 25, Drenaje principal; 26, Yacimientos arqueológicos; 27, Conjuntos arqueológicos del Paleolítico medio en superficie; 28, Carreteras; 29, Poblaciones.

Figure 9. Distribution of the surficial archaeological occurrences on the geological and geomorfological units. Geomorphological and geological map based on Benito-Calvo and Pérez-González (2015); archaeological occurrences based on Navazo (2006) Legend: 1, carbonates (Jurassic); 2, limestones, conglomerates and sandstones (F. Weald, Early Cretaceous); 3, sands, gravels and clays (facies Utrillas, Early Cretaceous); 4, carbonates with local flint nodules (Early Cretaceous); 5, conglomerates, sands, silts and clays (Oligocene-Miocene); 6, marls, evaporites and clays (facies Dueñas and Villatoro, Early Miocene); 7, limestone layer (Early Miocene); 8, conglomerates, sands and clays (facies Pedraja, Tierra de Campos, Santa María del Campo, Middle Miocene, Astaracian); 9, limestone with metric nodules of flint (Middle Miocene, Astaracian); 10, marls, limestones and clays (facies Dueñas, Late Miocene); 11, Lower páramo limestones (Late Miocene); 12, Lower Pleistocene fluvial terraces; 13, Middle Pleistocene fluvial terraces; 14;Upper Pleistocene fluvial terraces; 15, Upper PleistoceneHolocene fluvial terraces; 16, floodplain and valley beds; 17, alluvial cones; 18, semi-endorheic areas with seasonal pools; 19, alluvial-colluvial cones; 20, landslides; 21, colluvial deposits; 22, dolines; 23, faults; 24, anticline; 25, main drainage network; 26, archaeological sites; 27, Middle Palaeolithic archaeological occurrences; 28, roads; 29, populations. 
Benito-Calvo, A., et al., 2018. Evolución geodinámica pleistocena del valle del río Arlanzón... Boletín Geológico y Minero, 129 (1/2): $59-82$

En relación con otras zonas de la Meseta Ibérica, las tasas de incisión calculadas en el río Arlanzón presentan ciertas similitudes con las calculadas en el valle del Tajo, lo que podría indicar un levantamiento isostático conjunto, pero sin embargo, difieren notablemente de las calculadas en los Arribes del Duero. En los Arribes del Duero se proponen tasas altas de 2$3 \mathrm{~mm} / \mathrm{a}$ para los últimos 100 ka (Antón et al., 2012), mientras que en la depresión del Tajo, Silva et al. (2013), estiman tasas de incisión de $0.15 \mathrm{~m} / \mathrm{ka}$ para el Pleistoceno inferior, entre 0.09 y $0.05 \mathrm{~m} / \mathrm{ka}$ para el tránsito Pleistoceno inferior-medio, de $>0.1 \mathrm{~m} / \mathrm{ka}$ para el Pleistoceno medio final (en relación con procesos de subsidencia en yesos), y de $0.2 \mathrm{~m} / \mathrm{ka}$ para el Pleistoceno superior, en relación con los eventos climáticos Heinrich. En el valle bajo del río Tajo las tasas de incisión oscilan entre los 0.06-0.89 m/ka para periodos situados entre el Pleistoceno medio final y el Holoceno (Cunha et al., 2008).

Las cronologías y tasas de incisión en el Arlanzón informan sobre el periodo de formación entre los niveles de base que forman este tipo de terrazas, pero dadas las limitaciones que presenta el amplio error que las dataciones proporcionan en periodos antiguos, no se tienen datos directos de la duración de la formación de cada terraza individual, o sus tasas de agradación. No obstante, observaciones indirectas parecen indicar que el periodo de formación de cada terraza representaría un orden de magnitud mucho menor que el periodo entre cada terraza (del orden de 190-290 ka para los periodos entre las terrazas T3-T4T5 del Arlanzón). Por ejemplo, las cronologías de las Ilanuras aluviales actuales de la Península Ibérica muestran que se han desarrollado durante el Holoceno (Pérez-González et al., 2008; Thorndycraft and Benito, 2006), y además, los datos numéricos de terrazas recientes del Pleistoceno superior, sugieren su correlación con periodos climáticos de duración muy delimitada (eventos Heinrich, Pérez-González et al., 2008). Estos datos sugieren, por tanto, que la formación de las terrazas podría producirse en cortos periodos de tiempo, por ejemplo del orden o menores de $10 \mathrm{ka}$.

\section{Morfogénesis cuaternaria y relación con la formación de yacimientos arqueológicos.}

La incisión del valle medio del río Arlanzón y sus fases de agradación han controlado las líneas de construcción del paisaje en el entorno de la Sierra de Atapuerca, condicionando la formación del sistema kárstico que contiene los yacimientos de interior (Ortega et al., 2014; Arsuaga et al., 2014; Bermúdez de Castro et al., 2013; Carbonell et al., 2008), así como los asentamientos prehistóricos al aire libre documentados en el entorno de dicha sierra (Navazo and Carbonell, 2014; Navazo et al., 2011; Navazo, 2006).

\section{Relación de los niveles de base regionales con el sistema kárstico de la Sierra de Atapuerca}

La Sierra de Atapuerca es un relieve anticlinal, de tendencia NO-SE, formado mayormente en la serie carbonatada del Cretácico superior (Benito-Calvo and Pérez-González, 2015; Pérez-González et al., 1999; Pineda and Arce, 1997). En las calizas y dolomías del borde sudoeste de este anticlinal se desarrolla un sistema kárstico multinivel y cortical, cuyos rellenos contienen el registro arqueopaleontológico de la Sierra de Atapuerca (Ortega et al., 2014; PérezGonzález, 2001). El sistema endokárstico de la Sierra de Atapuerca está definido tres niveles o pisos principales de galerías subhorizontales paragenéticas (o formadas por disolución antigravitativa), cuya geometría indica la presencia de niveles freáticos cuaternarios de flujos SO-NE (desde el valle del Arlanzón hasta el río Pico), con un gradiente muy bajo o subhorizontal (Ortega et al., en este volumen; Ortega, 2009). La escasa pendiente y geometría de los niveles freáticos sugieren su correlación con niveles de base regionales situados a nivel, o ligeramente por encima. Los niveles de base regionales representan fases de estabilidad en la evolución del relieve, que habrían favorecido la perduración de los niveles freáticos en la misma posición y durante un periodo de tiempo necesario para generar las disoluciones kársticas subhorizontales. Como ya se ha expuesto, este periodo de tiempo en el caso de los niveles de base de las terrazas fluviales, podría representar periodos relativamente cortos (del orden o inferiores a $10 \mathrm{ka}$ ), según indican las cronologías de desarrollo de los planos aluviales actuales o los eventos de formación atribuidos a terrazas bajas del Pleistoceno superior en otras áreas (Pérez-González et al., 2008; Thorndycraft and Benito, 2006).

Los niveles freáticos de los pisos del sistema kárstico de la Sierra de Atapuerca, se localizan a 1015-1022 (nivel superior), 1000-1003 (nivel intermedio) y 985990 (nivel inferior) m s.n.m., que, respectivamente, indican cotas relativas medias respecto al río Arlanzón de +88, +68 y +51-58 m (Ortega et al., 2013; Ortega, 2009). El piso kárstico superior presenta una superposición de varios niveles freáticos que generan grandes volúmenes kársticos y que indican la persistencia de niveles freáticos con muy poca variación vertical a lo largo del tiempo (Ortega, 2009). Esta circunstancia coincide con lo observado en el valle del 
río Arlanzón en una posición adyacente a la Sierra de Atapuerca, donde en una posición equivalente al piso kásrtico superior, se desarrolla la terrazaT2 (+82-91 m) casi a nivel de la superficie de erosión SE4/PI. Esto indicaría muy bajas tasas de incisión en esta zona y la persistencia de niveles de base en una posición semejante desde la formación de dicha superficie de erosión hasta la terrazaT2 (+82-91 m), que explicarían la densa sucesión de morfologías freáticas que se observan en las galerías de este piso kárstico, así como las grandes dimensiones de estas cavidades. Aunque aún no se disponen de edades numéricas de la superficie SE3-PI, depósitos de piedemonte anteriores a los sistemas escalonados fluviales, posteriores a la colmatación de la cuenca miocena, se sitúan alrededor de 2.2 Ma hacia el este de la zona de estudio, en el borde norte de la sierra de Cameros (yacimiento plioceno de Villarroya, Pueyo et al., 1996). Por otro lado, la terraza T2 $(+82-91 \mathrm{~m})$, anterior a la terraza T3 $(+70-78 \mathrm{~m})$, y a los rellenos kársticos del nivel intermedio (Ortega et al., 2014; Carbonell et al., 2008), se situaría con una edad mínima de 1.13-1.22 Ma. De este modo, un posible rango de formación para los freáticos registrados el piso kárstico superior quedaría establecido entre 2.2 y 1.14-1.22 Ma, y durante periodos con polaridad inversa, como indica la magnetoestratigrafía de sedimentos arcillosos de posible origen paragenético medidos en este piso (Parés et al., 2016). Con posterioridad a la formación de la terraza T2 (+82-91 m), la incisión del río Arlanzón y la consiguiente incisión vadosa en el sistema kárstico posiblemente se realizaría con tasas de incisión $<0.038 \mathrm{~m} / \mathrm{ka}$, acorde con la tendencia observada en los patrones de incisión (Fig. 8), menor en los periodos más antiguos.

El piso o nivel kárstico intermedio ha sido correlacionado con el nivel de base de la terraza T3 $(+70-78$ m) (Ortega, 2009; Ortega et al., 2013). Como ya se ha adelantado, la edad de este piso se situaría plausiblemente entre 1.1-1.2 Ma, como indican la edad estimada para la terraza (1.14 $\pm 0.13 \mathrm{Ma}$, Moreno et al., 2012), y las edades más antiguas de los sedimentos contenidos en dicho piso kárstico (Parés et al., 2016; Ortega et al., 2014), cifradas hasta ahora entre los $1.13 \pm 0.18$ y $1.22 \pm 0.16$ Ma para la base de la secuencia alóctona de Elefante (Carbonell et al., 2008). Las tres edades están dentro del mismo rango de error estadístico, que abarca periodos tan amplios que resultarían suficientes para englobar la fase de agradación de la terraza fluvial y formación del piso kárstico intermedio (posiblemente $\leq 10 \mathrm{ka}$ ), así como las primeras fases vadosas en Elefante y Dolina.

Con posterioridad a la formación de la T3 $1+70-78$ $\mathrm{m})$, se produce la incisión del río Arlanzón, con tasas calculadas en $0.038 \mathrm{~m} / \mathrm{ka}$ (T3-T4). Esta incisión controlaría el encajamiento progresivo vertical vadoso que se observa actualmente en la parte inferior de los yacimientos de Elefante y Dolina, hasta la siguiente estabilización del nivel de base con la agradación de la terraza T4.

El piso kárstico inferior presenta techos a cotas variables entre $+51-58 \mathrm{~m}$, que han sido correlacionadas con los niveles de base de las terrazas T4 y T5 (Ortega et al., en este volumen; 2013), desarrolladas aproximadamente entre los 0.855-0.666 ka, en el tránsito Pleistoceno inferior-medio (Fig. 8). Los datos existentes dentro del piso kárstico intermedio también apuntan su formación en el tránsito Pleistoceno inferior-medio (Ortega et al., en este volumen; 2013). Así, en las cavidades del piso inferior se han citado polaridades inversas en sedimentos arenosos (Sima de los Huesos, Arnold et al., 2014), y polaridades normales en facies fluviales finas situadas a techo de arenas y gravas cuarcíticas (Cueva del Silo), que representan una antigua entrada directa del río Arlanzón. Como ya apuntó Ortega (2009), la posición relativa de estas facies fluviales alóctonas indica que dicha entrada que podría tratarse de un evento fluvial relacionado con el nivel de base T6 (+44-46 m), o más antigua. Al mismo tiempo que se desarrolla el piso inferior, se estarían formado facies de interior en la Galería Baja y la Galería del Silo (Ortega et al., en este volumen; 2013), y continuaría la sedimentación alóctona en Elefante y Dolina; todos ellos lugares donde está registrado el cambio Matuyama-Brunhes (Parés y Pérez-González, 1999; Parés et al., 2006, 2013; Parés et al., 2016).

Durante el Pleistoceno medio, se produce la excavación progresiva del río Arlanzón a razón de 0.061 $\mathrm{m} / \mathrm{ka}$ (Fig. 8). En este proceso se genera un valle encajonado en las facies arcillosas y margosas miocenas, que aparentemente desconectan los niveles freáticos fluviales de los carbonatos mesozoicos de la Sierra de Atapuerca, ya que no se reconocen niveles kársticos por debajo. A consecuencia de ello, cesarían las surgencias kársticas en la cabecera del río Pico (Ortega et al., en este volumen; 2013), provocando la disminución de las tasas de incisión en este valle secundario, desde evoluciones parecidas al Arlanzón, hasta los 0.01-0.02 m/ka estimados para los últimos $350 \mathrm{ka}$.

\section{Relación con los asentamientos paleolíticos al aire libre}

Las prospecciones realizadas en el entorno de la Sierra de Atapuerca han proporcionado evidencias de amplias zonas de concentración de conjuntos líticos 
prehistóricos (Navazo, 2006). Estos hallazgos corresponden principalmente a a conjuntos tecnológicos del Paleolítico medio y denotan la reiterada ocupación del paisaje en torno al valle del río Pico (Navazo and Carbonell, 2014), y, en cierto modo, delimitado por los valles de los ríos Arlanzón y Vena (Navazo, 2006).

Estas concentraciones líticas, interpretadas como asentamientos al aire libre, se disponen en la superficie del terreno, tanto sobre formaciones cuaternarias como sobre la superficie del sustrato pre-cuaternario (Navazo, 2006; Navazo et al., 2008, Mosquera et al., 2007, Navazo and Carbonell; 2014). La asociación que presentan los conjuntos líticos respecto a los nuevos datos cartográficos de las formaciones geológicas y geomorfológicas se puede observar espacialmente en la Fig. 9 y estadísticamente en la Fig. 10.

Además de ocupaciones localizadas en depósitos de terra rossa asociado a dolinas, y en relación con afloramientos de nódulos cretácicos de sílex (27-30 ka, Paleolítico medio-superior, yacimiento del Valle de las Orquídeas; Mosquera et al., 2007), las mayores concentraciones arqueológicas en superficie aparecen en terrenos sobre depósitos del Mioceno inferior y medio, y sobre formaciones cuaternarias de conos, coluviones, fondos de valle y terrazas medias y bajas (Figs. 9 y 10). Esta distribución está fuertemente con-

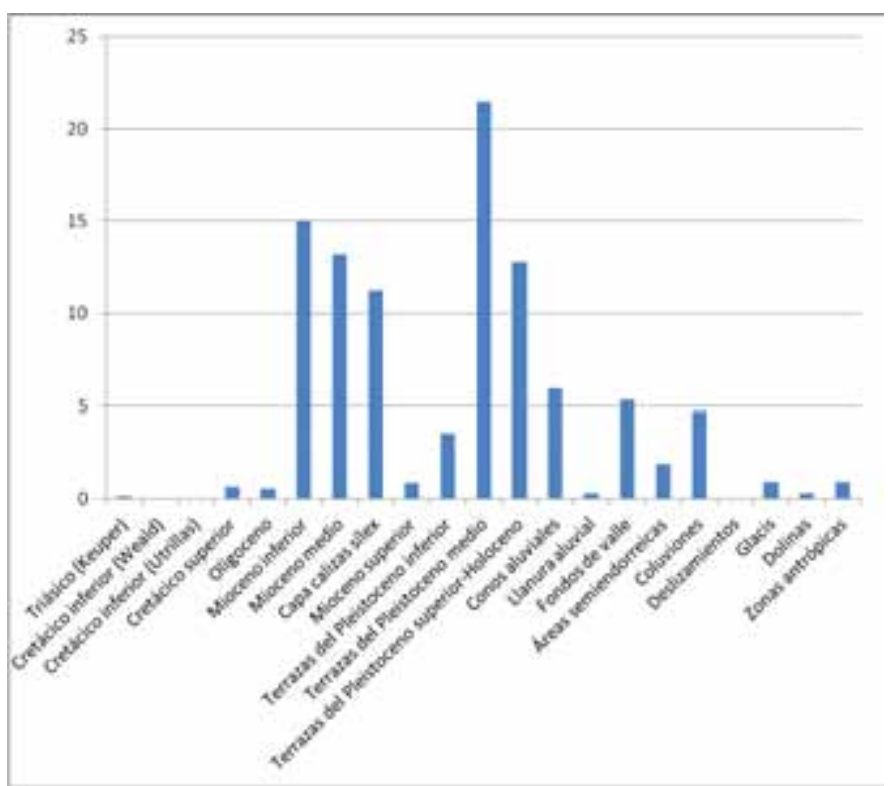

Figura 10. Distribución porcentual del material arqueológico superficial del Paleolítico medio, en función de las unidades geológicas y geomorfológicas.

Figure 10. Percentual distribution of Middle Palaeolithic archaeological occurrences on the geological and geomorfological units. dicionada por la existencia a techo de las unidades del Mioceno medio de la capa de calizas astaraciences (Figs. 9 y 10). Esta capa calcárea contiene nódulos métricos de sílex, que constituyen la principal fuente de materia prima identificada en dichos asentamientos al aire libre (Navazo et al., 2008a, Navazo et al., 2008b). Dicha formación geológica comenzó a ser exhumada durante el Pleistoceno inferior-medio, dando lugar a un plano resistente (plano de VillalbalRubena), que queda elevado sobre los valles circundantes del orden de $+40-90 \mathrm{~m}$ y en donde los procesos de meteorización han liberado bloques y fragmentos de sílex. Además, la erosión de estas calizas durante su exhumación, y sobre todo en fases posteriores del Pleistoceno medio y superior ha generado fragmentos de sílex que se disponen en posiciones topográficas inferiores de los valles del Arlanzón y del Pico, tanto sobre las facies del Mioceno medio e inferior, como en las formaciones geomorfológicas situadas ladera abajo, compuestas principalmente por conos aluviales y glacis que desaguan en los planos de las terrazas del Pleistoceno medio y superior (Figs. 9 y 10). De esta forma, la superficie de estas formaciones cuaternarias y sus escarpes coluvionados contendrían sílex, ya fragmentados, que constituirían áreas óptimas de aprovisionamiento de las poblaciones paleolíticas (Navazo, 2006).

Los conjuntos líticos de sílex no sólo se encuentran en superficie, sino que también aparecen englobadas en dichos depósitos cuaternarios. Las excavaciones realizadas hasta el momento en los yacimientos de Hundidero y Hotel California (HC) muestran distintos niveles arqueológicos con conjuntos musterienses elaborados principalmente en sílex néogeno procedente de la capa de calizas astarecienses (Navazo et al., 2008a, Navazo et al., 2008b, Navazo et al., 2011). Hundidero se ubica en un área semi-endorreica desarrollada sobre la terraza T4 (+60$67 \mathrm{~m}$ ) (Fig. 9), donde se ha observado una secuencia de gravas y arcillas, procedente de la degradación de dicha terraza por procesos de escorrentía. En estos depósitos se han excavado cuatro niveles arqueológicos estimados entre los 70 y los $50 \mathrm{ka}$ (TL and OSL, Laboratorio de Datación y Radioquímica de la Universidad Autónoma de Madrid; Navazo et al., 2011), que indican visitas repetidas durante los estadios MIS 4 y MIS 3.

Por otro lado, HC está compuesto por $1.2 \mathrm{~m}$ de arcillas arenosas, y clastos de cuarcita y sílex, en ocasiones flotando en las arcillas. En este yacimiento se separan hasta siete fases sedimentarias, con procesos de iluviación y tonos gleicos, que se desarrollan al pie del escarpe de un glacis de superficie erosiva localizado en la margen izquierda del valle del río 
Pico. Lateralmente, estos glacis del valle del río Pico presentan una cobertera de arcillas y clastos procedente de la erosión de las terrazas fluviales del Pleistoceno inferior del valle del Arlanzón (Fig. 9), y también de las laderas en el Mioceno, donde se encuentra el nivel calcáreo con sílex. En base a dicha posición geomorfológica y las características sedimentológicas de los sedimentos, los depósitos de HC son interpretados como un depósito coluvionar procedente del desmantelamiento episódico de la cobertera del glacis por procesos de escorrentía superficial y flujos gravitacionales. Al igual que en Hundidero, la secuencia estratigráfica de $\mathrm{HC}$ parece situarse desde el MIS4 hasta el MIS3, con cronologías de 71.0 $\pm 5.6 \mathrm{ka}$ para la base de la secuencia y $48.2 \pm 3.9 \mathrm{ka}$ hacia techo (SG-OSL, Arnold et al., 2013). Entre los años 2012 y 2017 se excavó otro yacimiento, Fuente Mudarra, situado en el mismo contexto geomorfológico que HC (Fig. 9).

\section{Conclusiones}

A partir de la colmatación y apertura de la cuenca neógena, los procesos exorreicos de incisión y vaciado han dominado la dinámica de construcción del paisaje en el sector NE de la cuenca del Duero. En este ámbito, se desarrolla el valle del río Arlanzón cuya dirección quebrada está controlada por lineaciones del sustrato, a favor de las cuales se ha excavado un valle de geometría asimétrica. La construcción del valle consta de 14 niveles de terrazas fluviales, compuestas preferentemente por facies de canales de gravas. Estas terrazas fluviales, datadas por distintos métodos entre el Pleistoceno inferior y el Holoceno, habrían representado etapas de duración relativamente corta, con niveles de bases estables y desarrolladas preferentemente en relación con estadios isotópicos fríos. Durante el Pleistoceno inferior, T3 $(+70-78 \mathrm{~m})$ y T4 $(+60-67 \mathrm{~m})$, parecen relacionarse con el MIS 34 y el MIS 22 respectivamente. En el Pleistoceno medio, T5 $(+50-58 \mathrm{~m})$ coincide con el MIS 16 , y los niveles T9 $(+35-37 \mathrm{~m})$ y T8 $(+28-32 \mathrm{~m})$, muy próximos entre sí, se asocian al MIS 10. En el límite Pleistoceno medio-superior, el nivel T11 (+12-14 m) aparece relacionado al MIS 6. La agradación de las terrazas está separada por periodos más dilatados, donde las tasas máximas de incisión desarrolladas en el valle principal, van incrementándose a lo largo del Pleistoceno y Holoceno. Las tasas estimadas se sitúan en $0.038 \mathrm{~m} / \mathrm{ka}$ para el tramo final del Pleistoceno inferior (T3-T4), $0.045 \mathrm{~m} / \mathrm{ka}$ en el tránsito entre el Plesitoceno inferior-medio, entre 0.061-0.185 m/ka durante el Pleistoceno medio y alrededor de 0.081 $\mathrm{m} / \mathrm{ka}$ en el Pleistoceno superior. Las mayores tasas se registran en el Holoceno. Esta dinámica muestra una aceleración general de la incisión que podría relacionarse con el levantamiento tectónico regional, quizás debido a ajustes isostáticos.

Esta dinámica ha controlado la formación del sistema endokárstico de la Sierra de Atapuerca, donde los conductos freáticos subhorizonaltes podrían haberse formado en periodos relativamente cortos, en relación con la agradación de las terrazas fluviales desarrolladas entre el Pleistoceno inferior y las fases inciales del Pleistoceno medio (T2-T5). Por otro lado, los volúmenes de encajamiento vadoso del sistema endokártico se habrían desarrollado en periodos dilatados, controlados por tasas de incisión fluvial bajas, con valores entre $<0.038 \mathrm{~m} / \mathrm{ka}$ y $0.045 \mathrm{~m} / \mathrm{ka}$. Durante gran parte del Pleistoceno inferior (periodo desde SE4 hasta T2), los bajos ratios de incisión habrían mantenido los niveles de base en posiciones semejantes en el entorno de la Sierra de Atapuerca, lo que podría haber condicionado las geometrías del piso kárstico superior, donde se observan grandes volúmenes causados por una sucesión de varias morfologías freáticas muy próximas.

En el exterior, las concentraciones de conjuntos arqueológicos aparecen asociados a áreas de captación de materias primas expuestas por procesos de meteorización, y de incisión y retrabajamiento durante el cuaternario que generan depósitos de sílex secundarios. En especial, la incisión de los valles a partir del tránsito Pleistoceno inferior-medio exhumó una capa de calizas neógenas con sílex, cuya erosión y resedimentación por procesos aluviales y gravitacionales en las vertientes de los valles generó grandes áreas de captación de materias primas durante el Pleistoceno medio y superior. Estos procesos están registrados en las secuencias de los yacimientos excavados, que representan áreas de degradación de depósitos cuaternarios preexistentes durante el MIS 4 y MIS 3.

\section{Agradecimientos}

Este trabajo ha estado financiado por los proyectos de investigación CEN001B10-2 de la Junta de Castilla y León, y GL2012-38434-C03-02, CGL2015-65387-C3-3$P$ y CGL2015-65387-C3-1-P del MINECO. Igualmente agradecemos al Equipo de Investigación de Atapuerca el apoyo logístico y científico. A Ana Álvaro Gallo por el análisis DRX en el Laboratorio de Arqueometría (CENIEH). A Leticia Miguéns Rodríguez y Javier Iglesias Cibanal por el análisis de textural en el Laboratorio de Muestras, Separación Mineral y 
Sedimentología del (CENIEH). Ana Isabel Ortega estuvo contratada con el Programa Juan de la Cierva (JCl2010-06792), y actualmente dispone de un contrato a cargo del proyecto CGL2009-1273-C03-01 del MINECO. Agradecemos a Eneko Iriarte y un revisor anónimo sus útiles comentarios y sugerencias.

\section{Referencias}

Alonso-Gavilán, G., Armenteros, I., Carballeira, J., Corrochano, A., Huerta, P. and Rodríguez, J.M. 2004. Cuenca del Duero. In: Vera, J.A. (ed.), Geología de España. Sociedad Geológica de España, IGME, Madrid, pp. 550-556.

Antón, L., Rodés, A., De Vicente, G., Pallàs, R., GarciaCastellanos, D., Stuart, F.M., Braucher, R. and Bourlès, D. 2012. Quantification of fluvial incision in the Duero Basin (NW Iberia) from longitudinal profile analysis and terrestrial cosmogenic nuclide concentrations. Geomorphology, 165-166, 50-61.

Arnáez, J. 1987. Formas y procesos en la evolución de vertientes de la Sierra de la Demanda. Colegio Universitario de La Rioja, Cuadernos de Investigación Geográfica, Logroño.

Arnold, L.J., Demuro, M., Navazo, M., Benito-Calvo, A. and Pérez-González, A. 2013. OSL dating of the Middle Palaeolithic Hotel California site, Sierra de Atapuerca, north-central Spain. Boreas, 42, 285-305.

Arnold, L.J., Demuro, M., Parés, J.M., Arsuaga, J.L., Aranburu, A., Bermúdez de Castro, J.M. and Carbonell, E. 2014. Luminescence dating and palaeomagnetic age constraint on hominins from Sima de los Huesos, Atapuerca, Spain. Journal of Human Evolution, 67, 85-107.

Arsuaga, J.L., Martínez, I., Arnold, L.J., Aranburu, A., GraciaTéllez, A., Sharp, W.D., Quam, R.M., Falguères, C., Pantoja-Pérez, A., Bischoff, J., Poza-Rey, E., Parés, J.M., Carretero, J.M., Demuro, M., Lorenzo, C., Sala, N., Martinón-Torres, M., García, N., Alcázar De Velasco, A., Cuenca-Bescós, G., Gómez-Olivencia, A., Moreno, D., Pablos, A., Shen, C.-C., Rodríguez, L., Ortega, A.l., García, R., Bonmatí, A., Bermúdez De Castro, J.M. and Carbonell, E. 2014. Neandertal roots: Cranial and chronological evidence from Sima de los Huesos. Science, 344, 1358-1363.

Benito, A., Pérez-González, A., and Santonja, M. 1998. Terrazas rocosas, aluviales y travertínicas del valle alto del río Henares. Geogaceta, 24, 55-58.

Benito, A. 2004. Análisis geomorfológico y reconstrucción de paleopaisajes neógenos y cuaternarios en la Sierra de Atapuerca y el valle medio del río Arlanzón. Tesis Doctoral. Universidad Complutense de Madrid, Madrid, $381 \mathrm{pp}$.

Benito, A. and Pérez-González, A. 2005. Las superficies erosivas de los páramos en el sector NE de la Cuenca del Duero y sus implicaciones en la conexión Duero-Bureba. Boletín Geológico y Minero, 116, 351-360.

Benito-Calvo, A. and Pérez-González, A. 2007. Erosion surfa- ces and Neogene landscape evolution in the NE Duero Basin (north-central Spain). Geomorphology, 88, 226-241.

Benito-Calvo, A., Pérez-González, A. and Parés, J.M. 2008. Quantitative reconstruction of Late Cenozoic landscapes: A case study in the Sierra de Atapuerca (Burgos, Spain). Earth Surface Processes and Landforms, 33, 196-208.

Benito-Calvo, A., Pérez-González, A., Magri, O. and Meza, P. 2009. Assessing regional geodiversity: the Iberian Peninsula. Earth Surface Processes and Landforms, 34, 1433-1445.

Benito-Calvo, A. and Pérez-González, A. 2015. Geomorphology of the Sierra de Atapuerca and the Middle Arlanzón Valley (Burgos, Spain). Journal of Maps $11,535-544$.

Benito-Calvo, A., Ortega, A.I., Pérez-González, A., Campaña, I., Bermúdez de Castro, J.M., Carbonell, E., 2017. Palaeogeographical reconstruction of the Sierra de Atapuerca Pleistocene sites (Burgos, Spain). Quaternary International 433, 379-392.

Bermúdez de Castro, J.M., Martinón-Torres, M., Blasco, R., Rosell, J. and Carbonell, E. 2013. Continuity or discontinuity in the European Early Pleistocene human settlement: The Atapuerca evidence. Quaternary Science Reviews, 76, 53-65.

Boquera, J., Gil, G. and Zubieta, F. 1978. Mapa Geológico de España, E 1:50.000, Hoja no 239 (Pradoluengo), Serie Magna. IGME, Madrid.

Bridgland, D. and Westaway, R. 2008. Climatically controlled river terrace staircases: A worldwide Quaternary phenomenon. Geomorphology, 98, 285-315.

Cabrera, R., Crespo, J.L., García, J.I., Mediavilla, B. and Armenteros, I. 1997. Mapa Geológico y Minero de Castilla y León, escala 1:400.000. Junta de Castilla y León. Sociedad de explotación e Investigación Minera de Castilla y León, S.A. (SIEMCALSA), Valladolid.

Carbonell, E., Bermúdez de Castro, J.M., Parés, J.M., PérezGonzález, A., Cuenca-Bescos, G., Ollé, A., Mosquera, M., Huguet, R., Made, J. van der, Rosas, A., Sala, R., Vallverdú, J., García, N., Granger, D.E., Martinón-Torres, M., Rodríguez, X.P., Stock, G.M., Vergès, J.M., Allué, E., Burjachs, F., Cáceres, I., Canals, A., Benito, A., Díez, C., Lozano, M., Mateos, A., Navazo, M., Rodríguez, J., Rosell, J. and Arsuaga, J.L. 2008. The first hominin of Europe. Nature, 452, 465-469.

Colchen, M. 1970. Géologie de la Sierra de la Demanda: Burgos-Logroño (Espagne). Faculté des Sciences de Paris, Paris. 524 pp.

Cunha, P.P., Martins, A.A., Huot, S., Murray, A. and Raposo, L. 2008. Dating the Tejo river lower terraces in the Ródão area (Portugal) to assess the role of tectonics and uplift. Geomorphology, 102, 43-54.

García, A., Cabra, P. and Solé, J. 1997. Mapa Geológico de España, E 1:50.000, Hoja no 238 (VillagonzaloPedernales), Serie Magna. IGME, Madrid.

García, J.M. 1979. El glaciarismo cuaternario en la Sierra de la Demanda (Prov. de Logroño y Burgos, España). Cuadernos de investigación: Geografía e historia, 5 (2), 3-26. 
Karampaglidis, T., Benito Calvo, A., Pérez-González, A., Baquedano, E. and Arsuaga, J.L. 2011. Secuencia geomorfológica y reconstrucción del paisaje durante el Cuaternario en el Valle del río Lozoya (Sistema Central, España). Boletín de la Real Sociedad Española de Historia Natural, 105, 149-162.

Lemartinel, B. 1985. L evolution morphologique de la Demanda et de ses piémonts durant le Néogéne. Revue Géographique des Pyrénées et du Sud-Ouest, 56, 471-490.

Lisiecki, L.E., and Raymo, M.E. 2005. A Pliocene-Pleistocene stack of 57 globally distributed benthic 180 records. Paleoceanography, 20, 1-17.

López-Recio, M., Silva, P.G., Roquero, E., Cunha, P.P., Tapias, F., Alcaraz-Castaño, M., Baena, J., Cuartero, F., Morín, J., Torres, T., Ortiz, J.E., Murray, A.S. and Buylaert, J.P. 2015. Geocronología de los yacimientos achelenses de Pinedo y Cien Fanegas (Valle delTajo) e implicaciones en la evolución fluvial en el entorno de Toledo (España). Estudios Geológicos, 71, 1-19.

Mediavilla, R.M., Dabrio, C.J. and Martín-Serrano, A. and Santisteban, J.I. 1996. Lacustrine Neogeno systems of the Duero Basin: evolution and controls. In: Friend, P.F., Dabrio, C.J. (eds.), Tertiary Basins of Spain: The Stratigraphic Record of Crustal Kinematics. Cambridge University Press, Cambridge, pp. 228-236.

Moreno, D. 2011. Datation par ESR de quartz optiquement blanchis (ESR-OB) de la région de Atapuerca (Burgos, Espagne). Application au site préhistorique de Gran Dolina (contexte karstique) et aux systèmes fluviatiles quaternaires de I Arlanzón e I Arlanza. Universitat Rovira i Virgili, Muséum National D Historie Naturelle, Tarragona. 282 pp.

Moreno, D., Falguères, C., Pérez-González, A., Duval, M., Voinchet, P., Benito-Calvo, A., Ortega, A.I., Bahain, J.J., Sala, R., Carbonell, E., Bermúdez de Castro, J.M. and Arsuaga, J.L. 2012. ESR chronology of alluvial deposits in the Arlanzón valley (Atapuerca, Spain): Contemporaneity with Atapuerca Gran Dolina site. Quaternary Geochronology, 10, 418-423.

Mosquera, M., Ollé, A., Pérez-González, A., Rodríguez, X.P., Vaquero, M., Verges, J.M. and Carbonell, E. 2007. Valle de las Orquídeas: un yacimiento al aire libre del Pleistoceno superior en la Sierra de Atapuerca (Burgos). Trabajos de Prehistroria, 64, 143-155.

Navazo, M., 2006. Sociedades cazadoras-recolectoras en la Sierra de Atapuerca durante el Paleolítico medio: patrones de asentamiento $y$ estrategias de movilidad. Tesis Doctoral. Universidad de Burgos, Burgos, 513 pp.

Navazo, M., Colina, A., Domínguez-Bella, S. and BenitoCalvo, A. 2008a. Raw stone material supply for Upper Pleistocene settlements in Sierra de Atapuerca (Burgos, Spain): flint characterization using petrographic and geochemical techniques. Journal of Archaeological Science, 35, 1961-1973.

Navazo, M., Colina, A., Domínguez, S. and Benito, A. 2008b. Caracterización y gestión de materias primas en los asentamientos al aire libre de la Sierra de Atapuerca (Burgos). In: Rovira Llorens, S., García-Heras, M., Gener, M., Montero, I. (eds.), Actas Del VII Congreso Ibérico de Arqueometría. Quadro, Madrid, 346-359.
Navazo, M., Alonso-Alcalde, R., Benito-Calvo, A., Díez, J.C., Pérez-González, A. and Carbonell, E. 2011. Hundidero: Mis 4 open air neanderthal occupations in Sierra de Atapuerca. Archaeology, Ethnology and Anthropology of Eurasia, 39, 29-41.

Navazo, M. and Carbonell, E. 2014. Neanderthal settlement patterns during MIS 4-3 in Sierra de Atapuerca (Burgos, Spain). Quaternary International, 331, 267-277.

Núñez, A., Colondrón, I., Zazo, C., Goy, J.L., Cabañas, I. and Uralde, M.A. 1975. Mapa Geológico de España, E 1:50.000, Hoja no 275 (Santa María del Campo), Serie Magna. IGME, Madrid.

Olivé, A., Ramírez, J.I., Ortega, L.I., Hernández, A. and Alvaro, M. 1990. Mapa Geológico de España, E 1:50.000, Hoja no 201 (Belorado), Serie Magna. IGME, Madrid.

Ortega, A.I. 2009. La evolución geomorfológica del karst de la Sierra de Atapuerca (Burgos) y su relación con los yacimientos pleistocenos que contiene. Tesis Doctoral. Universidad de Burgos, Burgos, $761 \mathrm{pp}$.

Ortega, A.I., Benito-Calvo, A., Pérez-González, A., Martín Merino, M.A., Pérez-Martínez, R., Parés, J.M., Aramburu, A., Arsuaga, J.L., Bermúdez de Castro, J.M. and Carbonell, E. 2013. Evolution of multilevel caves in the Sierra de Atapuerca (Burgos, Spain) and its relation to human occupation. Geomorphology, 196, 122-137.

Ortega, A.I., Benito-Calvo, A., Pérez-González, A., Carbonell, E., Bermúdez de Castro, J.M. and Arsuaga, J.L. 2014. Atapuerca Karst and its Palaeoanthropological Sites. In: Gutiérrez, F., Gutiérrez, M. (eds.), Landscapes and Landforms of Spain, Word Geomorphological Landscapes. Springer, 101-111.

Ortiz, J.E., Torres, T., Delgado, A., Reyes, E. and DíazBautista, A. 2009. A review of the Tagus river tufa deposits (central Spain): age and palaeoenvironmental record. Quaternary Science Reviews, 28, 947-963.

Panera, J., Torres, T., Pérez-González, A., Ortiz, J.E., RubioJara, S. and Uribelarrea, D. 2011. Geochronology of the Arganda Complex Terrace in the Jarama river valley (Madrid, Spain). Estudios Geológicos, 67, 495-504.

Parés, J.M. and Pérez-González, A. 1999. Paleomagnetic age for hominid fossils at Atapuerca Archaeological site, Spain. Science, 269, 830-832.

Parés, J.M., Pérez-González, A., Rosas, A., Benito, A., Bermúdez de Castro, J.M., Carbonell, E. and Huguet, R. 2006. Matuyama-age lithic tools from the Sima del Elefante site, Atapuerca (northern Spain). Journal of Human Evolution, 50, 163-169.

Parés, J.M., Arnold, L., Duval, M., Demuro, M., PérezGonzález, A., Bermúdez de Castro, J.M., Carbonell, E. and Arsuaga, J.L. 2013. Reassessing the age of Atapuerca-TD6 (Spain): New paleomagnetic results. Journal of Archaeological Science, 40, 4586-4595.

Parés, J.M., Ortega, A.I., Benito-Calvo, A., Aranburu, A., Bermúdez de Castro, J.M. and Carbonell, E. 2016. Paleomagnetic constraints on the Atapuerca Karst development (N Spain). In: Feinberg, J., Gao, Y., Calvin Alexandre, E. (eds.), Caves and Karst Across Time, Geological Society of America Special Paper 516, p. 285-300.

Pavón , J., García , J.M., Colmenero , J.R., Sánchez, L. and 
Manjón, M. 1973. Mapa Geológico de España, E 1:50.000, Hoja $n^{O} 237$ (Castrojeriz), Serie Magna. IGME, Madrid.

Pérez, B., Amezua, L., Miguel, I. and Morales, J. 2001. La fauna del yacimiento turoliense de Tariego de Cerrato (Palencia, Castilla-León). Revista Española de Paleontología $n^{\circ}$ extraordinario, 133-135.

Pérez-González, A., Gallardo-Millán, J.L., Uribelarrea, D., Panera, J. and Rubio-Jara, S. 2013. The MatuyamaBrunhes reversal at the river Jarama sequence of terraces between Velilla de San Antonio and Altos de la Mejorada, SE of Madrid (Spain). Estudios Geológicos, 69, 35-46.

Pérez-González, A., Martín-Serrano, A. and Pol, C. 1994. Depresión del Duero. In: Gutiérrez, M. (Ed.), Geomorfología de España. Rueda, Alcorcón, pp. 351-388.

Pérez-González, A., Parés, J.M., Gallardo, J., Aleixandre, T., Ortega, A.I. and Pinilla, A. 1999. Geología y estratigrafía del relleno de Galería de la Sierra de Atapuerca (Burgos) In: Carbonell, E., Rosas, A., Díez, J.C. (eds.), Atapuerca: Ocupaciones Humanas Y Paleoecología Del Yacimiento de Galería. Junta de Castilla y León, Conserjería de Educación y Cultura, Valladolid, pp. 31-42.

Pérez-González, A., Parés, J.M., Carbonell, E., Aleixandre, T., Ortega, A.I., Benito, A. and Martín, M.A. 2001. Géologie de la Sierra de Atapuerca et stratigraphie des remplissages karstiques de Galería et Dolina (Burgos, Espagne). L Anthropologie, 105, 27-43.

Pérez-González, A., Rublo Jara, S., Panera, J. and Uribelarrea, D. 2008. Geocronología de la sucesión arqueoestratigráfica de Los Estragales en la Terraza Compleja de Butarque (Valle del río Manzanares, Madrid). Geogaceta, 45, 39-42.

Pineda, A. 1996. El enlace y la paleogeografía neógena entre las Cuencas del Duero y del Ebro en la Bureba (Burgos). Boletín Geológico y Minero, 107, 14-28.
Pineda, A. 1997. Mapa Geológico de España, E 1:50.000, Hoja nO 167 (Montorio), Serie Magna. IGME, Madrid.

Pineda, A. and Arce, J.M. 1997. Mapa Geológico de España, E 1:50.000, Hoja no 200 (Burgos), Serie Magna. IGME, Madrid.

Pueyo, E., Muñoz, A. and Parés, J.M. 1996. Magnetoestratigrafía preliminar de los materiales plicoenos de la Cubeta de Villarroya (Sierra de Cameros, La Rioja). Geogaceta, 20, 1029-1032.

Santisteban, J.I., Mediavilla, R., Martín-Serrano, A. and Dabrio, C.J. 1996. The Duero Basin: a general overview. In: Friend, P.F., Dabrio, C.J. (eds.), Tertiary Basins of Spain: The Stratigraphic Record of Crustal Kinematics. Cambridge University Press, Cambridge, pp. 183-187.

Silva, P., Roquero, E., López-Recio, M., Huerta, P. and Tapias, F. 2013. Statistical approach to the chronosequence of fluvial terraces in the Tagus and Duero Basins (Central Spain). In: Baena, R., Fernández, J.J., Guerrero, I. (eds.), El Cuaternario Ibérico: Investigación en el S.XXI, VIII Reunión Del Cuaternario Ibérico, Sevilla-La Rinconada. AEQUA, Camas, Sevilla, pp. 29-33.

Thorndycraft, V.R. and Benito, G. 2006. The Holocene fluvial chronology of Spain: Evidence from a newly compiled radiocarbon database. Quaternary Science Reviews, 25, 223-234.

Zazo, C. and Goy, J.L. 1977. Geomorphological study of the confluence of the rivers Pisuerga, Arlanza, and Arlanzón, Basin of Duero (Spain). Presented at the $X$ Inqua Congress.

Zazo, C., Goy, J.L. and Hoyos, M. 1983. Estudio geomorfológico de los alrededores de la Sierra de Atapuerca (Burgos). Estudios Geológicos, 39, 179-185.

Zazo, C., Goy, J.L. and Hoyos, M. 1987. Contexto geológico y geomorfológico. In: Aguirre, E., Carbonell, E., Bermúdez de Castro, J.M. (eds.), El Hombre Fósil de Ibeas y El Pleistoceno de La Sierra de Atapuerca. Junta de Castilla y León, Valladolid, pp. 41-46.

Recibido: marzo 2015

Revisado: mayo 2015

Aceptado: septiembre 2015

Publicado: marzo 2018 